1 Running Head: eDNA Biodiversity Map of California

2 Title: A Biodiversity Composition Map of California Derived from Environmental DNA

3 Metabarcoding and Earth Observation

4 Authors: Meixi Lin ${ }^{1}$, Ariel Levi Simons ${ }^{2}$, Emily E. Curd ${ }^{1}$, Ryan J. Harrigan ${ }^{1}$, Fabian D.

5 Schneider ${ }^{3}$, Dannise V. Ruiz-Ramos ${ }^{4,5}$, Zack Gold $^{1}$, Melisa G. Osborne ${ }^{6}$, Sabrina Shirazi ${ }^{7}$, Teia

6 M. Schweizer ${ }^{1,8}$, Tiara N. Moore ${ }^{1,9}$, Emma A. Fox $^{1}$, Rachel Turba ${ }^{1}$, Ana E. Garcia-Vedrenne ${ }^{1}$,

7 Sarah K. Helman ${ }^{1}$, Kelsi Rutledge ${ }^{1}$, Maura Palacios Mejia ${ }^{1}$, Miroslava N. Munguia Ramos ${ }^{1}$,

8 Regina Wetzer ${ }^{10,11}$, Dean Pentcheff ${ }^{10,11}$, Emily Jane McTavish ${ }^{5}$, Michael N. Dawson ${ }^{5}$, Beth

9 Shapiro $^{7,12}$, Robert K. Wayne ${ }^{1}$, Rachel S. Meyer ${ }^{1,7^{*}}$

$10 *$ Corresponding Author: E-mail: rsmeyer@ucla.edu

$11{ }^{1}$ Department of Ecology and Evolutionary Biology, University of California-Los Angeles, Los

12 Angeles, CA 90095, USA

$13{ }^{2}$ Department of Marine and Environmental Biology, University of Southern California, Los

14 Angeles, CA 90089, USA

$15{ }^{3}$ Jet Propulsion Laboratory, California Institute of Technology, 4800 Oak Grove Drive, Pasadena,

16 CA 91009, USA

$17{ }^{4}$ U.S. Geological Survey, Columbia Environmental Research Center, Columbia, MO 65201,

18 USA

$19{ }^{5}$ Department of Life \& Environmental Sciences, University of California-Merced, Merced, CA

2095343, USA 
$21{ }^{6}$ Department of Molecular and Computational Biology, University of Southern California, Los

22 Angeles, CA 90089, USA

$23{ }^{7}$ Department of Ecology and Evolutionary Biology, University of California-Santa Cruz, Santa

24 Cruz, CA 95064, USA

$25{ }^{8}$ Department of Biology, Colorado State University, Fort Collins, CO 80523, USA

$26{ }^{9}$ School of Environmental and Forestry Sciences, University of Washington, Seattle, WA 98195,

27 USA

$28{ }^{10}$ Research and Collections, Natural History Museum of Los Angeles County, Los Angeles, CA

2990007, USA

$30{ }^{11}$ Biological Sciences, University of Southern California, Los Angeles, CA 90089, USA

$31{ }^{12}$ Howard Hughes Medical Institute, University of California-Santa Cruz, Santa Cruz, CA 95064,

32 USA 


\section{A Biodiversity Composition Map of California Derived from Environmental DNA}

\section{Metabarcoding and Earth Observation}

\section{$35 \underline{\text { Abstract }}$}

Unique ecosystems globally are under threat from ongoing anthropogenic environmental

37 change. Effective conservation management requires more thorough biodiversity surveys that

38 can reveal system-level patterns and that can be applied rapidly across space and time. We offer

39 a way to use environmental DNA, community science and remote sensing together as methods to

40 reduce the discrepancy between the magnitude of change and historical approaches to measure it.

41 Taking advantages of modern ecological models, we integrate environmental DNA and Earth

42 observations to evaluate regional biodiversity patterns for a snapshot of time, and provide critical

43 community-level characterization. We collected 278 samples in Spring 2017 from coastal, shrub

44 and lowland forest sites in California, a large-scale biodiversity hotspot. We applied gradient

45 forest to model 915 family occurrences and community composition together with environmental

46 variables and multi-scalar habitat classifications to produce a statewide biodiversity-based map.

4716,118 taxonomic entries recovered were associated with environmental variables to test their

48 predictive strength on alpha, beta, and zeta diversity. Local habitat classification was diagnostic

49 of community composition, illuminating a characteristic of biodiversity hotspots. Using gradient

50 forest models, environmental variables predicted $35 \%$ of the variance in eDNA patterns at the

51 family level, with elevation, sand percentage, and greenness (NDVI32) as the top predictors.

52 This predictive power was higher than we found in published literature at global scale. In

53 addition to this indication of substantial environmental filtering, we also found a positive

54 relationship between environmentally predicted families and their numbers of biotic interactions.

55 In aggregate, these analyses showed that strong eDNA community-environment correlation is a 
56 general characteristic of temperate ecosystems, and may explain why communities easily

57 destabilize under disturbances. Our study provides the first example of integrating citizen science

58 based eDNA with biodiversity mapping across the tree of life, with promises to produce large

59 scale, high resolution assessments that promote a more comprehensive and predictive

60 understanding of the factors that influence biodiversity and enhance its maintenance.

61 Keywords: environmental DNA; DNA metabarcoding; California; citizen and community

62 science; ecological modeling; gradient forest; remote sensing; community ecology

63 
$\underline{\text { Introduction }}$

Global biodiversity is currently undergoing rapid loss (Pimm et al. 2014, Ceballos et al. 2015, Díaz et al. 2019) with many species and geographic areas (Myers et al. 2000) threatened by unique environmental challenges such as climate change and habitat degradation. The scientific community needs better tools to provide critical baseline biodiversity data that can be applied rapidly with minimal cost and effort (Bush et al. 2017). A recently discussed goal of biodiversity conservation is to estimate the Essential Biodiversity Variables (Pereira et al. 2013)

71 that are a minimal set of fundamental observations needed to support multi-purpose, long-term

72 planning at various scales, from genetic variation, to species diversity and ecosystem structure.

73 However, scaling up from in situ biological measures remains challenging (Pereira et al. 2013).

74 Changes in the richness and turnover of species are usually tracked in taxonomically or

75 spatiotemporally restricted studies because technical feasibility limits large scale monitoring

76 (Cristescu 2014). Very few studies attempt to assess the complex composition of entire

77 biological communities (Karimi et al. 2018, George et al. 2019).

As one new approach to achieving this need for tracking biodiversity over large scales,

79 the past decade has showcased the growing power of biodiversity inventory by non-experts: the use of technology assisted citizen and community science (CCS). CCS for traditional

81 biomonitoring through observation has already dramatically scaled up biomonitoring efforts

82 (Theobald et al. 2015, Kobori et al. 2016, Waller 2019). However, these approaches favor

83 diurnal macroscopic taxa and often omit cryptic and microbial taxa (Theobald et al. 2015).

84 Recently, programs have launched that arm community scientists with the capacity to sample

85 environmental DNA (eDNA) from their surroundings (Biggs et al. 2015, Miralles et al. 2016,

86 Deiner et al. 2017, Meyer et al. 2019) which can be probed for nearly any taxonomic group using 
87 metabarcoding methods (Bohmann et al. 2014, Deiner et al. 2016, Thompson et al. 2017,

88 Taberlet et al. 2018, Franklin et al. 2019). These methods are improving in accuracy as public

89 DNA sequence databases grow, while simultaneously decreasing in cost, suggesting community-

90 powered eDNA surveys may provide the more complete biodiversity data needed for mapping

91 and predicting taxon presence as well as determining community assemblage patterns and the

92 Essential Biodiversity Variables.

Multi-locus metabarcoding of eDNA from surface soil and sediment retains a record of

94 taxa recently present in the local area. The temporal span of the surface DNA record from these

95 substrates may be weeks to months, even several years (Andersen et al. 2012, Parducci et al.

96 2017). Surface DNA can be easily collected with minimal environmental impact (Meyer et al.

97 2019) and the taxonomic signatures preserved in eDNA perform similarly to direct observation

98 surveys (Tedersoo et al. 2014, Lejzerowicz et al. 2015) including vertebrate and vascular plant

99 communities that are of conservation concern, but also yield a species catalog that is much

100 broader, including bacteria and archaea, often-overlooked meiofauna, protozoans, non-vascular

101 plants, algae, and fungi.

102 Efforts to integrate surface eDNA with remote measures of ecosystem properties present

103 an opportunity to improve models and ecological theories (Yamasaki et al. 2017). In parallel

104 with the developments in eDNA metabarcoding on biodiversity monitoring, on the ground and

105 space-based technologies yield increasingly copious and accessible abiotic data (Pettorelli et al.

106 2014, Schimel et al. 2019) on land cover, topography, soil property (e.g. US Geological Survey),

107 bioclimate (Fick and Hijmans 2017), human impact (WCS and CIESIN 2005) and vegetation

108 (e.g. Sentinel-2; European Space Agency) which can be used to characterize eDNA biodiversity

109 changes across a landscape (van den Hoogen et al. 2019, Crowther et al. 2019). Biotic-abiotic 
110 interactions among soil properties (e.g. $\mathrm{pH}$ and nutrient availabilities), climate, plant coverage,

111 and habitat type affect soil alpha and beta diversity (Fierer and Jackson 2006, Ranjard et al. 2013,

112 Montagna et al. 2018, George et al. 2019). Different taxonomic groups have differential

113 responses to environmental cues in diverse ecosystems from tropical mountains to temperate

114 ecosystems (Thompson et al. 2017, Karimi et al. 2018, Montagna et al. 2018, Peters et al. 2019).

115 For example, a national-scale soil eDNA survey in England showed that animal and microbial

116 richness responded to different environment factors but beta-diversity trends were shared across

117 taxonomic groups (George et al. 2019). However, biodiversity rich regions and biodiversity

118 hotspots have scarcely been studied in taxonomic biodiversity-ecological response models. Such

119 regions are discontinuous in environmental clines and have high endemism (Myers et al. 2000,

120 Thompson et al. 2017). Surface eDNA signatures could aid predictions of regional biodiversity

121 patterns to guide conservation planning (Bush et al. 2017, Jetz et al. 2019, Breed et al. 2019) and

122 theory development (Konopka 2009, Kurtz et al. 2015, Prosser 2015, Koskella et al. 2017).

123 To integrate the regional environmental variables for predictions on eDNA biodiversity

124 turnover, joint-species distribution models are needed that can utilize big-data. The gradient

125 forest model is an offshoot of the random forest algorithm (Breiman 2001) that attempts to

126 explain turnover in community assemblages and can be used for regional community turnover

127 predictions (Ferrier and Guisan 2006, Ellis et al. 2012). Briefly, the gradient forest first

128 establishes a composite relationship of species' response (in this case, eDNA profiles) to

129 environmental predictors, and then projects the turnover of this response across unsampled

130 regions using this relationship (Ellis et al. 2012). The gradient forest is robust, performs well

131 with large number of correlated explanatory variables and captures non-linear responses of

132 community turnover (Fitzpatrick and Keller 2015). It also handles the input of biotic matrix 
133 without the need for ordination, thus utilizing all information available in modeling (Ellis et al.

134 2012, Pitcher et al. 2012). However, such integration of remotely sensed variables with eDNA

135 for biodiversity mapping remains unexplored.

136 Here, we present an analysis of eDNA-based biodiversity from the California

137 Environmental DNA (CALeDNA; Meyer et al. 2019) program and propose a framework to

138 perform citizen and community science enabled biodiversity mapping using surface eDNA

139 profiles and environmental variables. It is the first test case of an eDNA survey to describe,

140 analyze and predict the biotic composition of a large-scale biodiversity hotspot, the state of

141 California (CA). Launched in 2017, CALeDNA volunteers have contributed over 5000

142 georeferenced surface samples and have participated in the evaluation of presence patterns

143 through online tools (www.ucedna.com). Results from 278 samples collected in one seasonal

144 window largely from natural reserves are presented here and integrated multiple Earth

145 observations in diversity analyses and models to explain habitat diversity and community

146 turnover. Our research demonstrates how large-scale surface collections can be used for routine

147 mapping of biodiversity, which may help track the resilience of species and community

148 responses to environmental fluctuations and anthropogenic threats.

$149 \underline{\text { Methods }}$

$150 \quad$ Sampling design

151 We aimed to sample biodiversity from a wide variety of habitats across the state of

152 California using directed volunteers and eDNA metabarcoding. CALeDNA scientists recruited

153 volunteers to perform sampling, and were guided through virtual and in-person training to select

154 soil and sediment sites from natural areas where permission was granted (Supplemental

155 Methods). Sampling steps were guided by a smartphone webform (Meyer et al. 2019) made in 
156 Kobo Toolbox (kobotoolbox.org). Surface samples were collected by filling $2 \mathrm{~mL}$ tubes with

157 substrate from $<2 \mathrm{~cm}$ depth in three biological replicates, each $30 \mathrm{~cm}$ apart. Volunteers changed

158 gloves between sampling sites. Samples were frozen at $-80{ }^{\circ} \mathrm{C}$ immediately upon their return to

159 CALeDNA headquarters at UC Los Angeles.

160 To minimize the potential effect of seasonal variations in eDNA profiles, we selected

161 samples from March 2017 to July 2017. We classified the predominant biome using photographs

162 and a variety of geolocation data (Supplemental Methods; Table 1; Table S1). We selected 100

163 samples from each of three transect types which were coast, shrub/scrub (abbreviated as

164 "shrub"), and forest that covered the broadest latitudinal range possible. Samples with

165 ambiguous metadata were removed, resulting in a total of 278 samples (98 coast, 89 shrub and

16691 forest) used in subsequent analyses.

167 Compilation of environmental variables

168 We assembled environmental variables across six main categories: location, habitat,

169 bioclimate, topography, human impact and vegetation (Supplemental Methods; Table S1; Text

$170 \mathrm{~S} 1)$. All raster layers were aligned and projected to a unified $100 \times 100 \mathrm{~m}$ grid from Google

171 Earth Engine (Coordinate Reference System for this project: ESPG 4326, WGS84). Layers with

172 a higher original resolution were down-sampled using a mean aggregation method. Layers with a

173 lower or same original resolution were projected using bilinear method for continuous values and

174 nearest neighbor method for categorical values. Layers were stacked and clipped to California's

175 extent, and used for point extraction. For coastal sites outside of the raster's geographical

176 coverage, values were extracted by the closest point available in $0.5 \mathrm{~km}$ radius. If the closest

177 point fell outside of the radius, that site was assigned "NA" value. All computation and analyses 
178 were performed in R version 3.5.3 (R Core Team 2019). Raster operations were performed using

179 R package raster (Hijmans 2017).

180 Considering that many environmental variables are correlated, we evaluated the

181 Pearson's correlation coefficient of the 56 numerical environmental variables and hierarchically

182 clustered the variables according to the coefficients into variable groups using R functions cor,

183 hclust and cutree. To reduce collinearity and improve interpretability in community modeling,

184 we created a reduced set of numerical environmental variables that had an $\mathrm{R}^{2}<0.8$ (Table 1 ) for

185 downstream analysis.

186 DNA extractions, amplification and sequencing

187 DNA extraction, amplification and sequencing followed Curd et al. 2019. Briefly, three

$188250 \mathrm{mg}$ biological replicate soil samples from each site were fully homogenized and pooled per

189 site. DNA was extracted using QIAGEN DNeasy PowerSoil Kit (Qiagen, Valencia, CA, USA)

190 according to the manufacturer's instructions. Negative controls were included in every batch of

191 12-18 extractions. DNA was amplified by polymerase chain reaction (PCR), using primers for

192 five barcode regions: $16 S$ (515F and 806R; Caporaso et al. 2012), $18 S$ (Euk_1391f and EukBr;

193 Amaral-Zettler et al. 2009), CO1 (mlCOIintF and Fol-degen-rev; Yu et al. 2012, Leray et al.

194 2013), fungal ITS1 (“FITS”; ITS5 and 5.8S; White et al. 1990, Epp et al. 2012), and plant ITS2

195 ("PITS" ITS-S2F and ITS-S3R; Gu et al. 2013). For samples belonging to the coast transect, they

196 were additionally amplified using $12 S$ barcode targeting fish (MiFish; Miya et al. 2015) for

197 another study. Although the result is not described here, the raw sequence file contains those

198 sequences. Primer sequences and thermocycling profiles can be found in Table S2.1-2. All PCR

199 amplifications were performed in triplicate and with additional PCR negative controls. Triplicate

200 positive amplifications confirmed by gel electrophoresis, were pooled by sample and barcode to 
equimolar levels (Supplemental Methods), indexed and sequenced on an Illumina MiSeq v6 with a target sequencing depth of 50,000 reads/sample/metabarcode. Five of the 278 sites were processed as biological replicates by different technicians to inspect taxonomic variation in independent DNA extraction and technical processing.

\section{Bioinformatics and data processing}

We used default settings in the Anacapa Toolkit (Curd et al. 2019) for multi-locus sequence data processing and taxonomy assignment. In brief, quality control of raw sequences was performed using Cutadapt (Martin 2011) and FastX-Toolkit (Gordon et al. 2010), and inference of Amplicon Sequence Variants (ASVs) was made with DADA2 (Callahan et al. 2016).

211 Taxonomy assignment was made on each ASV using Bowtie2 (Langmead and Salzberg 2012)

212 and the Bayesian Lowest Common Ancestor algorithm (BLCA; Gao et al. 2017) on custom

213 metabarcode-specific reference databases, created using Creating Reference libraries Using

214 eXisting tools (CRUX; Curd et al. 2019). Bowtie2 first aligns ASVs against the corresponding

215 metabarcode-specific reference database and returns up to 100 alignments for each ASV. BLCA

216 then determines the lowest common ancestor (LCA) from Bowtie2 hits for each ASV and assigns

217 a bootstrap confidence for each level of the taxonomic path. Taxonomy assignments with a

218 bootstrap confidence cutoff score over 0.6 were kept for each ASV. ASVs with the exact same

219 inferred LCA passing confidence filter were summed into one taxonomic entry as the

220 species/phylotype/MOTU equivalent in this study and will be referred as "taxonomic entry" in

221 the following text.

222 To informatically control for contamination, we further removed all singleton or

223 doubleton ASVs, and ASVs that occurred more than or equal to three times in all blank samples 
224 from subsequent analyses (Table S3). Resulting ASVs from each primer were converted to

225 phyloseq objects using the R package ranacapa (Kandlikar et al. 2018). For subsequent alpha

226 and beta diversity analyses requiring rarefaction, we performed rarefaction in 10 replicates and

227 took the mean using the custom_rarefaction function in the R package ranacapa (Table S2.3;

228 Text S2). Reads with no assignment were not removed before rarefaction.

229 To evaluate how material aliquoted for DNA extraction and independent lab processing

230 influenced taxon profiles, we estimated concordance in the decontaminated and further rarefied

231 datasets between biological replicates (Text S3).

\section{Comparisons with Traditional Surveys}

233 To compare the eDNA taxonomic results to traditional surveys, we compared eDNA

234 results to the curated species inventory of the University of California Natural Reserve System

235 (UCNRS), which records Chordata, Arthropoda, and Streptophyta (see Supplemental Methods).

236 We counted how many taxon records were shared or unique to eDNA results or traditional

237 records (UCNRS) at classification levels of order, family and genus combining all reserves and

238 within each reserve.

239 We then developed a metric of traditional observation score (TOS) in eDNA taxonomic

240 assignment. TOS uses all species observation and collection records in the Global Biodiversity

241 Information Facility (GBIF) database from a broad region centered on California to score

242 whether the taxon assignment of an eDNA ASV has been observed. A TOS $>0$ suggests there is

243 support for the assignment of an ASV based on its presence in the TOS region (see Supplemental

\section{Methods).}




\section{Alpha Diversity}

Alpha diversity was calculated using Observed and Shannon's Diversity Index in R

247 package vegan (Oksanen et al. 2019) to compare categorical variable groups (details in

248 Supplemental Methods). The significance level was set at 0.05. The difference between each

249 category was tested using the Kruskal-Wallis Test with Bonferroni correction for multiple testing.

250 Post hoc analysis was performed using the Dunn Test (function dunnTest in R package FSA;

251 Ogle et al. 2019).

We evaluated the relationships of alpha diversity measures and the reduced set of 33

253 continuous environmental variables (Table 1) as well using individual linear regressions

254 (alpha_diversity variable) of alpha diversity measures and environmental variables using

255 function $l m$ in $\mathrm{R}$. To provide a more complete evaluation and account for the collinearity in the

25633 variables, we also used partial least square (PLS) models with methods adapted from Lallias

257 et al. (Lallias et al. 2015, George et al. 2019; see Supplemental Methods). PLS models are

258 designed to handle regressions that have many, possibly correlated, explanatory variables and

259 relatively few observations. We fit PLS models for alpha diversity in each metabarcode using

260 function $p l s r$ in R package $p l s$ (Mevik et al. 2019) with three components included in the model

$261($ ncomp $=3)$, and the variable importance in projection measure was calculated using function

262 VIP.R in R package $p l s$ extension (https://mevik.net/work/software/VIP.R).

\section{Beta Diversity}

264 Unless otherwise specified, all functions mentioned in this section are from R package

265 vegan. Community composition was visualized by plotting sample relative abundance of the top

266 ten phyla for metabarcodes $16 S, 18 S$, and CO1, and top ten classes for PITS and FITS, 
267 partitioned by the major habitat. This was implemented by function plot_taxa in R package 268 microbiomeSeq (Ssekagiri et al. 2017).

269 Composition was analyzed using unconstrained ordination. We calculated the binary

270 Jaccard dissimilarity distance from the rarefied dataset for each metabarcode dataset and

271 performed principal coordinate analysis (PCoA), permutational multivariate ANOVA

272 (PERMANOVA) analysis by function adonis with 2999 permutations, and tests for the

273 assumption of homogeneity of dispersion using the betadisp function (Supplemental Methods).

274 Permutest function was used to test for the significance, with 2999 permutations. P values were

275 adjusted using the Bonferroni method for both PERMANOVA and betadisp tests. To control for

276 the effect of spatial correlation, partial constrained analysis of proximities (CAP) was done for

277 the habitat and soil property variables while removing the effect of geographic coordinates.

278 Partition of variance was evaluated using the varpart function. To control for the large variation

279 in aquatic/coastal sites, PERMANOVA was repeated by excluding the samples from the coastal

280 sites. We also partitioned the data by the four categories in the majorhab variable (aquatic;

281 herbaceous; shrub and tree dominated habitats) and performed PCoA and PERMANOVA

282 analyses within each major habitat. Post hoc explanation of the ordination axes was performed

283 by fitting the reduced set of numerical variables (Table 1) onto the PCoA result using the envfit

284 and ordisurf functions (Supplemental Methods).

\section{Zeta Diversity}

To measure the fraction of unique categories of organisms held in common among

287 nearby communities, we set cluster size to 4 nearby sites (Supplemental Methods) and calculated

288 zeta four diversity $\left(\zeta_{4}\right)$. The value of $\zeta_{4}$ was scaled for each geographic cluster as a fraction of its

289 average taxonomic richness per sample $\left(\zeta_{1}\right)$ using the function Zeta.decline.ex in the R package 
ZETADIV (Latombe et al. 2018). We tested the likelihood of two models of community assembly, using the Akaike Information Criterion (AIC) score within ZETADIV, through calculations of

292 how zeta diversity decays with sampling order. Based on prior analyses (Hui et al. 2014) decays

293 which follow a power-law of the form $\zeta_{N}=\zeta_{1} N^{-b}$, or an exponential of the form $\zeta_{N}=\zeta_{1} e^{b .(N-1)}$,

294 were associated with a niche differentiation or stochastic process of community assembly,

295 respectively. Scaled $\zeta_{4}$ diversity values were then plotted on a map of California using the R 296 package Leaflet (Cheng et al. 2019).

Environmental factor groups were made by binning environmental variables according to

298 their categories (Table 1). To determine the variation in $\zeta_{4}$ diversity attributed to either

299 geographic distance or an environmental factor group, we used the function Zeta.varpart to

300 analyze generalized linear models (GLM) generated using the function Zeta.msgdm within the R

301 package ZETADIV. For each GLM we only used samples where both the geographic cluster ID

302 and particular environmental factors were known.

303

304

305

306

307

308

309

310

311 classification-tree based gradient forest model using default settings to the biological matrix

312 derived, but increased the number of trees to 2000 per family to increase the stability of the 
313 model (Breiman 2001). To assess model robustness and stability, we repeated the gradient forest

314 model 20 times with the same settings and recorded the families with positive $\mathrm{R}^{2}$ values, the

315 overall corrected $\mathrm{R}^{2}$, the importance of each explanatory variable. To assess model power and

316 reliability, we used a permutation approach akin to Bay et al. 2018: we randomized the predictor

317 matrix 100 times and ran the model (Supplemental Methods).

318 To visualize the community turnover gradient forest model over space, we used the input

319 of all 33 environmental variables from $100 \mathrm{~m}$ x $100 \mathrm{~m}$ grids in the extent of California without

320 extrapolation according to the gradient forest manual (Pitcher et al. 2011; Supplemental

321 Methods). We used the top three principal components from the transformed environmental

322 variables and visualized them by red, green and blue (RGB) bands (Ellis et al. 2012). Each

323 environmental variable loading was visualized by function biplot in R. To differentiate model

324 performance from the innate high-dimensional nature of the environmental variable matrix, we

325 scaled the environmental variables and performed the same PCA and visualization procedure

326 without using the model ("uninformed map") and performed a mantel test and a monotonic

327 regression between the biological matrix and either the uninformed map or gradient forest

328 informed map.

329 Network analysis

330 Results for each metabarcode were summarized by family, filtered on read depth and

331 frequency and used in SPIEC-EASI ecological co-occurrence network analysis using R package

332 SpiecEasi (Kurtz et al. 2015) for cross domain analysis that incorporates all five metabarcodes

333 into one complex network (see Tipton et al. 2018). Topological parameters were determined in

334 Cytoscape (v. 3.6.1; Shannon et al. 2003) using the NetworkAnalyzer tool. To observe the

335 relationship between network degrees and the prediction $\mathrm{R}^{2}$ of each family from gradient forest, 
an ordinary least squares (OLS) linear regression model was made using the $l m$ function in R and interactions were with R package Interactions (v. 1.1.1; Long 2019).

To evaluate the co-occurrence and gradient forest predictor patterns in a phylogenetic

339 framework, the 915 families used in the gradient forest modeling were mapped onto the Open

340 Tree of Life (tree.opentreeoflife.org) and a synthetic tree was generated using synthesis release

341 v12.3. Datasets were mapped next to the phylogeny tips using the Interactive Tree of Life

342 (https://itol.embl.de/).

$343 \underline{\text { Results }}$

344 Metabarcoding result summary

The 278 selected samples that citizen scientists collected between March 2017 to July

3462017 represented coast, shrub, and forest transects (Figure 1A; Table S1) and were sequenced to

347 capture diversity across kingdoms. Each metabarcode recovers their target groups as expected

348 (Table S2.1; Figure 1C), with $16 S$ amplifying Bacteria and Archaea, $18 S$ and CO1 broadly

349 amplifying eukaryotes including Animalia, Chromista, Fungi, Protozoa and some Plantae, ITS1

350 targeting fungi ('FITS') selecting for Ascomycota and Basidiomycota, and the ITS2 region

351 targeting plants ('PITS') predominantly selecting for Chlorophyta and Streptophyta.

352 The 278 samples, five 'biological replicate' samples processed in duplicate by different

353 technicians to assess the stability of results, and 21 negative controls as PCR blanks or extraction

354 blanks, were sequenced, amounting to 75,830,796 reads for the five metabarcoding loci (Table

355 S3). The average read depth per sample per locus was 54,554 reads. After several steps of quality

356 control implemented in the Anacapa Toolkit (Curd et al. 2019), taxonomic assignment to NCBI-

357 based reference databases, and subsequent sequence decontamination based on the negative

358 controls, a total of $16,157,425$ reads were assigned to 16,118 taxa from the five metabarcodes 
359 included in all transects. The median assigned read depth was 7,717 (Table S3; Figure S3) and 360 mean taxa identified was 778 per sample.

A small test on variation in the replicate extraction and processing showed our methods

362 were similar to what others have reported. An average of $97 \%$ taxonomic entries overlapped

363 when negative results were counted and $44 \%$ entries overlapped when only presence was

364 counted (Text S3; Table S4.2; Figure S4). Our eDNA metabarcoding results are sensitive to

365 stochastic amplification even in triplicate PCR (Shirazi et al. in prep) and DNA extraction

366 replicates may truly differ by small scale spatial heterogeneity (Lanzén et al. 2017). We

367 interpreted this to expect zero-enriched data and less power to predict taxon presence with eDNA.

368 To prepare results for alpha and beta diversity analyses, sequence rarefaction was used to

369 remove variation in sequencing depth. For the $16 S$ metabarcode, 210 samples were kept with

3702,000 reads/sample; for 18S, 221 samples were kept with 4,000 reads/sample; for CO1, 220

371 samples were kept with 1,000 reads/sample; for FITS, 225 samples were kept with 4,000

372 reads/sample; for PITS, 221 samples were kept with 1,000 reads/sample (Text S2; Table S2.3;

373 Figures S5,S6). Despite fairly deep sequencing, stringent sample filtration was necessary to meet

374 sufficiency metrics practiced by the metabarcoding community (Goldberg et al. 2016, Taberlet et

375 al. 2018).

376 In summary, assignments spanned 81 phyla with most reads and taxonomic entries being

377 assigned to Proteobacteria, Ascomycota and Basidiomycota (Figure 1B,C). The $16 S$ metabarcode

378 targeting prokaryotes generated most of the unique taxonomic entries (Table S3; Figure 1C). 


\section{Comparison with traditional surveys: eDNA results partially overlap with traditional}

observations

Our comparison between metabarcoding results from within the UCNRS and a curated species list of UCNRS Streptophyta, Arthropoda and Chordata made by traditional surveys (Table S5) showed partial overlap across multiple classification levels. Forty-four Streptophyta

384 families were only found in eDNA, 77 were only in traditional observations, 65 were recovered 385 from both methods. We found 110 Arthropoda families were only recovered from eDNA, 139 were only in traditional observations, and 16 were recovered from both methods. No Chordata families were jointly recovered from both methods (Table S5.1). These results suggest many families are more conducive to being detected by either eDNA or observation, and the overlap is substantial in plants but not in arthropods. We acknowledge the seasonality of the UCNRS observations was not known, and could include many species that would not be expected to occur in the seasonal window eDNA was sampled (Bolger et al. 2000). 


\section{Alpha diversity varies at the local scale and across the terrestrial-marine interface}

Three patterns emerged from alpha diversity Kruskal-Wallis tests of observed and

404 Shannon Index alpha diversity of rarefied metabarcode datasets. First, we found spatial

405 stratification for alpha diversity measures in the loc variable (name of places visited reported by

406 volunteers) and minorhab (minor habitat) variable for all metabarcodes besides $C O 1$, and

407 stratification for the clust variable (neighboring cluster of sites within a radius of $0.5 \mathrm{~km}$ derived

408 from GPS record) for $16 S$ and FITS (Figure 2A; Figure S7; Table S7), indicating bacterial and

409 fungal alpha diversity is locally constrained in California. Second, transect was not significant

410 for $18 S$ and PITS (Figure 2B; Figure S8) and major habitat (majorhab) was not significant in any

411 tests (Figure 2C; Figure S9). Land use classification (NLCD) and ecoregion, yielded mixed

412 results where Shannon and Observed tests conflicted in significance (Table S7). Post-hoc Dunn

413 tests of NLCD in $16 S$ and FITS metabarcodes showed that samples belonging to open water,

414 wetlands, and developed space were often part of the pairs that were different (Figure S10; Table

415 S7.3). Third, we found substrate type $(S o S)$ differed for all metabarcode tests except for $18 S$, and

416 post hoc tests showed sand samples were consistently lower in alpha diversity across

417 metabarcodes (Figure 2D; Figure S11) compared to soil and sediment. Soil class (taxousda) tests

418 showed no significance.

419 Individual linear regressions of alpha diversity statistics with continuous environmental

420 observations showed fungal organisms (FITS) were best predicted compared to other

421 metabarcodes, and five FITS models had an $\mathrm{R}^{2}>0.2$. Observed FITS alpha diversity with

422 greenness had the highest correlation $\left(\mathrm{R}^{2}=0.29\right.$; Table S8; Figure $\left.\mathrm{S} 12\right)$. In contrast, while a

423 second analysis to evaluate the combined effect of the 33 environmental variables using PLS

424 models also found the highest regression coefficients for FITS alpha diversity $\left(\mathrm{R}^{2}=0.17\right.$ and 
425 0.15), soil carbon content variable orcdrc, and aspect were the variables with the highest

426 importance (Table S9).

427 Beta diversity: Community composition varied by habitat characteristics

428 Bar plots showing relative abundance displayed similar patterns of variation across major

429 habitats in $16 S$ and FITS results (Figure S13A,D). Bar plots of all other metabarcodes showed

430 differences in composition and relative abundance profiles both within major habitats and

431 between aquatic and terrestrial habitats. Differences were especially prominent in aquatic

432 samples (Figure S13), where Ascomycota were largely absent and Chlorophyceae and

433 Ulvophyceae were frequently dominant (Figure S13C,E). Interestingly, some CO1 samples were

434 dominated by Bacteroidetes, one of the most dominant bacterial phyla in the ocean (Figure

435 S13C). We followed these observations of compositional diversity with several beta diversity

436 analyses.

437 In community dissimilarity analysis, beta dispersion testing showed significant

438 heterogeneity of multivariate dispersion (variance) within groups for all metabarcode and

439 category combinations except loc, majorhab, transect, and clust for the PITS metabarcode (Table

440 S10.1). PERMANOVA results showed significant differences among all different environmental

441 variable categories (Table S10.2; Figures S14-S17). Beta diversity was significantly different

442 across major habitat groups despite many overlapping sites in the ordination plots (Figure 3;

443 Figures S14-S17). Groups exhibited differences both in within-group variation (dispersion) and

444 in mean values of group centroids. In particular, samples from aquatic environments were more

445 dispersed in the ordination (Figure 3A). To examine if this dispersion was driven by coastal

446 samples that contain some community members restricted to marine systems, we removed

447 coastal sites and re-examined community clusters (Figure 3C,D). Samples still exhibited 
448 clustering by major habitat in PERMANOVA and beta dispersion tests (Table S10.3-4; Figure

449 3C; Figure S18), suggesting observed aquatic beta diversity was also driven by freshwater sites.

450 Minor habitat (minorhab) composition within each of the four major habitats was found

451 to contribute strongly to dissimilarity (PERMANOVA, adjusted $\mathrm{P}<0.01$ in 2,999 permutations;

452 Table S10.5-6). Jaccard dissimilarity PCoA revealed interesting features of beta diversity. In

453 aquatic habitats, lacustrine and riverine samples overlapped and were largely separate from

454 coastal minor habitats in all metabarcodes. The 'marine intertidal zone III' cluster separated from

455 other marine and estuarine groups in all metabarcodes except for $16 \mathrm{~S}$. In contrast, the $16 \mathrm{~S}$

456 community of 'estuarine bay tidal flat' was distinct from others when it was not for other

457 metabarcodes (Figure 4). In herbaceous-dominated habitats, grassland samples were less

458 dispersed in $16 S$ results than in other metabarcodes. In PITS results, minor habitat groups largely

459 overlapped, but in all other metabarcodes, grasslands were distinct in composition. Scrub

460 habitats were similar in $16 S$ and $\mathrm{CO} 1$ but distinct in FITS communities. Mixed chaparral and

461 coastal scrub overlapped in composition. In tree-dominated habitats, the coastal oak woodland,

462 which was the most heavily sampled (Table S1.3), was dispersed (Figure 4). Other minor

463 habitats formed smaller clusters, which were most separated from each other in the $16 S$

464 metabarcode results.

465 CAP analysis removing the effect of spatial autocorrelation found that samples clustered

466 tighter according to major habitat (Figure 3A,B; Figure S19). The unique contribution of major

467 habitat (adjusted $\mathrm{R}^{2}$ ranging from $2.14 \%$ (PITS) to $9.65 \%(16 S)$ ) is larger than the contribution of

468 local geographical categories (adjusted $\mathrm{R}^{2}<2.00 \%$, Table $\mathrm{S} 11$ ).

Envfit results showed that photosynthetic activities (NDVI32 and greenness) were most

470 highly correlated with $16 S, 18 S$ and FITS (Table 2; Table S12; Figure S20). Soil organic carbon 
471 content $(\mathrm{orcdrc})$ was most highly correlated with CO1, and Isothermality (bio3) was most highly

472 correlated with PITS. Only 24 of 165 tests did not display significant relationships between

473 variables and community composition. Bio14 and bio3 were the least frequently significant

474 variables (Table S12).

Zeta diversity species retention rate over space and composition predictability

483 for $18 S$, COI and FITS datasets, with the highest variance explained at $32 \%$ for the FITS dataset.

$48416 S$ and PITS datasets had low $\zeta_{4}$ predictabilities. Variables related to small-scale location

485 describe minimal variation $(<1 \%)$ in $\zeta_{4}$ diversity for communities (Table 3$)$. To better

486 understand spatial community stability, two models of zeta diversity decay were tested: the

487 power law model and the exponential model. The power law model was found to be a better fit

488 for communities described in all but the PITS metabarcode results, which followed the

489 exponential decay model, suggesting lower spatial autocorrelation in plant and algal

490 communities (Table S13; Figure S21).

\section{Gradient Forest: Mapping biodiversity turnover in California}

492 Our community mapping approach used a gradient forest model that inputs 272 sites x

493915 families as a response variable matrix and 272 sites x 33 "reduced" set of environmental 
494 variables as a predictor matrix (Table S14). The gradient forest model explained 35\% of

495 variation in the biotic matrix, and all 915 families were effectively modeled (i.e. had an $\mathrm{R}^{2}>0$ )

496 and had high stability across 20 replicated runs (Average $\mathrm{R}^{2}=0.349 \pm 0.0004$; Average families

497 effectively modeled $=915 \pm 0$; Table S15). Using a permutation approach, we confirmed the

498 mean overall $\mathrm{R}^{2}$ and number of families with positive $\mathrm{R}^{2}$ for true observations were higher than

499 all the permuted runs (Figure S22). Many of the most responsive families were from marine

500 aquatic sites, and some of these were low in observation frequency (Figure 5B; Figure S23).

501 However, we did not observe a relationship between the observation frequency of a family and

502 its gradient forest $\mathrm{R}^{2}$ (Figure S24), in contrast to what others have reported (Stephenson et al.

503 2018). This full gradient forest model showed elevation (elev), sand percentage (sndppt),

504 photosynthetic activities based on vegetation greenness (NDVI32) and temperature of the wettest

505 quarter (bio8) were the four most important predictors (Figure 5A) with a maximum of 0.018 in

506 importance (Figure 5A).

507 Gradient forest provides information on the rate of community turnover along

508 environmental gradients (Ellis et al. 2012). We plotted the relative density of splits and

509 cumulative importance for environmental variables. Within the top three environmental variables,

510 we found nonlinear community changes. For elevation, rapid community (high splits density)

511 turnover occurred at $0 \mathrm{~m}$ and above 1,000 $\mathrm{m}$ (Figure 5C,D). For sand percentage, important splits

512 were mainly distributed at 23\%,43\% and 74\% sand (local maxima with the highest density,

513 Figure 5C,D), which have similarity to the soil texture triangle in the USDA system

514 (Groenendyk et al. 2015). For photosynthetic activities (NDVI32), important splits were mainly

515 distributed along $-0.16,0.05$, and 0.28 (scale: -1 to 1 ; Figure 5C,D). 
Our map of California biodiversity resembled EPA North America Level II and

517 California Level III Ecoregion maps (U.S. Environmental Protection Agency 2010, 2012), which

518 were created with different input data and methods (Figure 6C-E). In the gradient forest map

519 (Figure 6A), the majority of central and southwestern CA community type (red) corresponded to

520 Mediterranean California (Figure 6C. pale green, Level II 11.1.), characterized by medium

521 photosynthetic activities (NDVI32), lower elevation (elev) and higher precipitation seasonality

522 (bio15). The northwestern CA community type (purple) overlapped with Marine West Coast

523 Forest (Figure 6C. blue, Level II 7.1.), characterized by a higher photosynthetic activity (NDVI32)

524 compared to Mediterranean California. The northeastern CA type (green) corresponded with the

525 Western Cordillera (Figure 6C. green, Level II 6.2.), with higher elevation (elev) and sand

526 percentage (sndppt). The southeastern CA type (orange) was characterized by warm deserts

527 (Figure 6C. yellow, Level II 10.2.), with lower elevation, almost no photosynthetic activity and

528 higher temperature in the wettest month (bio8).

We checked the model prediction robustness by regenerating the California map without

530 being informed by eDNA (Figure 6B), and these results were less visually similar compared with

531 the eDNA-informed map (Figure 6A) and other California published maps such as the EPA

532 North America Level II Ecoregion map (U.S. Environmental Protection Agency 2010, Omernik

533 and Griffith 2014; Figure 6C). This purely physical approach of community turnover mapping

534 showed adding eDNA improves gradient forest informed mapping limitedly by a $1.4 \%$ reduction

535 in stress performance statistics; 5\% increase in Mantel correlation $\mathrm{R}^{2}$ (Figure S25).

536 Because several of the most predicted families were marine, we generated a gradient

537 forest model without coastal sites to examine which environmental variables shifted in their

538 importance rank and to see how many families would still be modeled (meaning they transcend 
539 the coastal-fully terrestrial boundary). Without coastal sites, 802 families could still be

540 effectively modeled and these had similar distribution of $\mathrm{R}^{2}$. In the model without coastal sites,

541 we could still explain $30.4 \%$ of the variation in the biotic matrix. We observed the sharpest

542 increases in biol and human footprint $(h f p)$ importance, and a sharpest decreases in NDVI32

543 (where negative values correspond to water bodies; Weier and Herring 2000), greenness, and

544 coastal aerosol (BI) importance (Figure S26) when coastal sites were removed. Other

545 environmental variables were similar in importance and rank to the full gradient forest model.

\section{Ecological co-occurrence relates to gradient forest predictability}

Co-occurrence patterns reflect biotic niche processes that maintain biodiversity patterns.

548 We found a relationship between network degrees and family predictor $\mathrm{R}^{2}$ using an OLS linear

549 model, which indicates environmental filtering (Horner-Devine et al. 2007). A family-level co-

550 occurrence network produced 916 edges connecting 290 nodes (families) out of the total 304

551 families that met minimum frequency thresholds for analysis (Table S16; Figure 7A). The

552 maximum edges per node was 48. The number of edges (degrees) per node showed no

553 correlation with Neighborhood Connectivity (Pearson $r=0.058)$ or Radiality $(r=-0.113)$ but was

554 highly correlated with Stress $(r=0.900)$.

555 In the OLS linear model, interaction effects of site frequency were also considered.

556 Model results showed a modest positive relationship (Adj $\left.\mathrm{R}^{2}=0.22\right)$ between the number of

557 edges and $\mathrm{R}^{2}$ for families, indicating the families determined by gradient forest to be under the

558 most environmental filtering were also the families most integrated in ecological networks based

559 on their numbers of degrees. However, the interaction between frequency in sites and network

560 degrees was also significant $(\mathrm{P}<0.02$; Figure 7B). In a phylogenetic analysis of these patterns, we

561 observed that families with high network degrees and high gradient forest predictor values were 
562 widely distributed across clades and kingdoms, but heaviest in the clades containing the class

563 Flavobacteriia and the SAR supergroup (Figure 7C), suggesting these communities might have

564 lowest resilience under abiotic change and interacting partner drop out.

\section{Discussion}

We have entered a new era of biomonitoring where species observations by the public

567 outpace both field collections and on-the-ground observations made by scientists (Pearce-

568 Higgins et al. 2018). With eDNA as a CCS tool (Biggs et al. 2015, Miralles et al. 2016, Sutter

569 and Kinziger 2018), the public can be additionally armed with the capacity to inventory

570 taxonomically broad local biodiversity from a small soil sample collection. Soils and sediments

571 used in this study, collected by CCS volunteers, had an average of 778 taxonomic lineages

572 identified in each sample. Several programs have demonstrated successes with eDNA as a CCS

573 tool, and new innovations have made sampling easier for volunteers (e.g. Thomas et al. 2019)

574 but thus far, most public efforts have targeted only a handful of species (e.g. algae and fish;

575 JonahDNA.com). We demonstrate an approach using CCS collections obtained rapidly from a

576 broad area within a seasonal snapshot. eDNA from these collections is co-analyzed with

577 environmental data to explain and predict taxonomically broad biodiversity patterns, ultimately

578 producing fine-scale beta diversity maps, and providing data that can advance ecological theory.

579 Our study mapped environmental data with beta diversity of varied taxonomic groups

580 across California's ecoregions at a higher resolution than currently available in statewide maps

581 (Figure 6). Previous efforts have used climate and other abiotic data and models, and integrated

582 them with traditional observational records such as herbarium specimens (Baldwin et al. 2017) to

583 produce maps used to conserve threatened species (Jenkins et al. 2015), assess deforestation

584 (Zarnetske et al. 2019) and evaluate species richness and endemism (Baldwin et al. 2017). 
585 However, few mapping efforts coupled these data with remotely sensed variables such as from

586 the Sentinel-2 instrument and local-scale observations of taxonomy biodiversity such as from

587 eDNA. These additional measures enable community mapping at a finer grid size than $5 \mathrm{~km}$

588 (Jenkins et al. 2013, 2015, Pimm et al. 2014, Baldwin et al. 2017, Zarnetske et al. 2019). Fine-

589 scale resolution has been shown to be better at aligning on-the-ground measures of biodiversity

590 with spectral measures (e.g. Wang et al. 2018). As more relevant observations from space

591 become available for analysis, community composition and turnover predictability should

592 improve, which can help define areas that require distinct management (McKnight et al. 2007).

593 As a validation of our approach, our community turnover map aligned well at the

594 regional scale with the EPA Ecoregion level III map (Figure 6D), although this conclusion is

595 based only on a qualitative comparison. Nonetheless, there are fine-scale differences, such as the

596 community designation near the San Francisco Bay (USDA Forest Service 2007, U.S.

597 Environmental Protection Agency 2012). Our map is based on one season of eDNA observations

598 from 2017 and thus has newer data than the observations that contribute to the EPA and USDA

599 maps (Figure 6D,E). Such designation differences between maps suggest eDNA may detecting

600 annual to decadal habitat conversion (Kadir et al. 2013). Future work should include

601 comparisons of eDNA-enhanced maps from different seasons and years to assess community

602 stability and the sensitivity of eDNA to temporal change.

604 local endemism, and high human impact (Hopper 1979, Myers et al. 2000). However, only

605 recently with the advancement in DNA-based biomonitoring has species richness been

606 systematically tested in microbial groups (Deiner et al. 2017). Our metabarcoding results from

607 both macro- and microbial species confirm that richness exhibits local variation (Figure 2; Figure 
S7). Alpha diversity patterns observed in $18 S, C O 1$ and PITS are consistent with past

invertebrate, vertebrate and floristic community surveys that found richness does not vary according to mid-scale environmental partitions within a biodiversity hotspot (Pryke and

611 Samways 2009, Demarais et al. 2017; Table S7; Figure S9). We assessed which metabarcode’s

612 alpha diversity patterns could be modeled with environmental variables and found fungi (FITS)

613 were predicted by indices of photosynthetic activity greenness and NDVI32 (individual linear

614 models LM; Table S8; Figure S12), soil carbon and aspect (partial least square models PLS;

615 Table S9). These LM results suggest plant-soil reinforcement such as rhizosphere interactions

616 may drive fungal richness (Timling et al. 2014, Tedersoo et al. 2014, Prober et al. 2015, Yang et

617 al. 2017, Erlandson et al. 2018). We note indices of photosynthetic activity have not been

618 included as part of most microbiome studies (Karimi et al. 2018, Bahram et al. 2018, George et

619 al. 2019) so their importance is still being discovered. For the subset of studies we found that had

620 included NDVI as a predictor, it was found to be important in modulating soil fungal and

621 herbivore nematodes communities (Timling et al. 2014, Yang et al. 2017, van den Hoogen et al.

622 2019). We interpret the PLS results to indicate that soil carbon distribution in California is

623 largely connected to fungal activity such as from saprobes (Erlandson et al. 2018), globally

624 dominant fungal decomposers that assort into ecological guilds (Větrovský et al. 2019).

625 Latitude, temperature, precipitation and $\mathrm{pH}$ were not significant in alpha diversity

626 analysis (Tables S8,S9) although other studies have highlighted these factors as major correlates

627 of alpha diversity (Fierer and Jackson 2006, Tedersoo et al. 2014, George et al. 2019, Crowther

628 et al. 2019), explained by Rapoport's Rule (Stevens 1989). We have considered that this

629 discrepancy may be partially due to our omission of environmental extremes; for example, all

630 samples had near neutral pH values (pH: $\operatorname{Min}=5.05 ; \operatorname{Max}=8.50 ;$ Median =6.40; Table S1.4). 
631 However, such gradients have only been shown to affect certain taxa, and therefore the breadth

632 of phylogenetic biodiversity encapsulated in each metabarcode may impair the detection of

633 taxon-specific alpha diversity relationships. For example, while legumes (Garcillán et al. 2003)

634 exhibit a latitudinal gradient with richness, invertebrates do not (Kerr 1999) and both of these

635 groups are represented in the $18 S$ results.

636 Our beta diversity analyses show that most environmental categories can significantly

637 partition samples according to taxonomic composition (Table S10; Figures 3 and 4; Figures S14-

638 S17), suggesting that surface communities are largely filtered by ecology rather than neutral

639 processes (Bahram et al. 2018). These patterns remained significant after exclusion of the more

640 dispersed coastal sites and location effects (Table S10.3-4; Table S11; Figures S18,S19).

641 However, we found substantial overlap in community composition ordinations, as has been

642 shown in the global Earth Microbiome Project (Thompson et al. 2017) and regional soil

643 biodiversity ordination plots (George et al. 2019; Table S10; Figure 3). In our ordinations,

644 groups separated from each other when fine-scale categories are used, such as minor habitat

645 within partitioned major habitat, suggesting a large amount of community partitioning is

646 harbored within major habitats categories (Figure 4). We found prokaryotic diversity was

647 particularly diagnostic of minor habitats in ordinations (Figure 4A). California is known for

648 shifting ecotones that make management units unstable (e.g. Hennessy et al. 2018). We propose

649 community classifications that include prokaryotes could be developed from analysis of eDNA-

650 based composition and habitat features and these could serve as quantitative metrics to detect

651 sites transitioning from one microhabitat type to another (Warton et al. 2015).

652 Examination of community compositions in site clusters using zeta diversity found

653 evidence of high spatial decay in plants but not in other groups. Zeta $(\zeta)$ diversity is particularly 
654 useful for diagnosing local compositional variation. Compared with beta diversity that utilizes

655 pair-wise dissimilarity, $\zeta_{4}$ diversity partitions regional diversity (gamma diversity) in three or

656 more assemblages (Hui and McGeoch 2014; Figure S21A). Zeta diversity decay exhibits a power

657 law relationship with sampling order, whereas the PITS dataset of Chlorophyta and Streptophyta

658 is exponential (Table S13; Figure S21F). This observation suggests that plant-algal guilds are

659 stochastic across space and may be especially sensitive to microclimates and stratified local

660 nutrient availability as supported by previous studies (Sebastià 2007, Eisenlohr et al. 2013). We

661 suggest this pattern be more extensively examined in future soil eDNA inventories of the

662 kingdom Plantae to confirm if stochasticity is truly reflective of spatial arrangement or

663 influenced by the chance of detection with DNA-based methods. The latter could be biologically

664 dependent (e.g. presence of saprobes to process tissue and DNA), physically dependent (e.g. cell

665 shedding), or chemically dependent (e.g. suppression of amplifiable DNA by phytochemicals).

666 As an emerging field, we strongly encourage more attention on optimizing alignment between

667 plant eDNA $\zeta$ diversity and plant distribution on the landscape.

668 Environmental variables (Tables 2 and 3) can have power to predict general biotic

669 patterns and can illuminate possible drivers of community turnover (Figure S20) because they

670 can readily be compared across studies (Omernik and Griffith 2014). For example,

671 photosynthetic activities (NDVI32/greenness) had the highest correlation with the observed beta

672 diversity structure in bacteria (16S), eukaryotes (18S) and fungi (FITS) in the envfit analyses

673 (Table 2; Figure S20). Previous studies have supported a similar correlation of plant productivity

674 and microbial beta diversity in global drylands (Delgado-Baquerizo et al. 2016) and in Tibetan

675 Plateau (Yang et al. 2017). Isothermality (bio3) has strong positive associations with PITS

676 turnover, suggesting inland arid California regions with low isothermality display nestedness in 
677 the biodiversity encompassed by these markers, as has been shown with plants in Australia

678 (Gibson et al. 2012) and in South American seasonally dry forests (Silva and Souza 2018).

679 Organic carbon ( orcdrc) was strongly associated with CO1 community turnover, which mirrors

680 associations reported in soil macrofaunal communities, particularly nematodes (Jackson et al.

681 2019). Overall, zeta diversity largely support the envfit results, although zeta diversity had poorer

682 explanatory power for 165 patterns, which can be attributed to its greater sensitivity to common

683 groups (Table 3; Simons et al. 2019) such as the nearly ubiquitous taxa in Proteobacteria. being correlated with each other (Figure S1), joint statistical analysis of multiple environmental variables is attractive to model biodiversity. However, examination of single variables will remain important as new regions are added to CALeDNA surveys. For example, it is unclear whether any of these patterns hold in agricultural regions of California such as the Central Valley, where we have few CCS samples. Agriculture has been proposed to 'reset' many of these abiotic constraints (Li et al. 2020). Single variables will also remain important to compare studies, given

691 there is no standard set of variables. The use of Essential Biodiversity Variables could better

692 enable comparative study of joint multivariable predictions of beta diversity (Jetz et al. 2019).

694 or global coverage, family-level taxa presence could be strongly predicted and used to produce

695 community turnover maps (Figures 5 and 6). Both the full gradient forest model and the model

696 without coastal sites show that there are no predictors that are substantially stronger than others

697 (Figure 5A; Figure S26A). Our full gradient forest model is in agreement with many proposed

698 patterns of how the environment filters communities. Elevation (elev), sand percentage (sndppt),

699 photosynthetic activities (NDVI32) and mean temperature in the wettest quarter (bio8) were the 
among the most important predictors (Figure 5A) and all of these variables had been proposed to

701 be prominent drivers in community structures worldwide. Similarly, microbial turnover patterns

702 had been linked to elevational gradients (Collins et al. 2018, Peters et al. 2019) and to particle

703 size fractions (Sessitsch et al. 2001, Ehrlich et al. 2015). Our analysis complements such findings

704 of variation in the rate of compositional turnover along environmental gradients, which have

705 been the focus of many traditional ecology case studies. For example, fast community turnover

706 was proposed at $0 \mathrm{~m}$ elevation (Figure 5C), recapitulating the observation that communities are

707 highly structured and patchy in these coastal areas, which was also suggested by the envfit results

708 to $B 1$ (coastal aerosol).

709 Finally, we suggest eDNA ecological network analyses should be leveraged so that the

710 biotic interaction dependence can be contrasted with dependence or sensitivity to the abiotic

711 environment. Our work shows a positive relationship between the number of degrees a family

712 has and its propensity for environmental filtering based on gradient forest predictability, and

713 shows there is phylogenetic conservation of this positive relationship (Figure 7). These results

714 suggest that climate change and other disturbance can lead to community network dropout and

715 cause a cascade of community change that may not be anticipated. Other studies focused on a

716 single kingdom have come to similar conclusions, such as in microbial variation in an altitudinal

717 gradient in the Atacama Desert, Chile (Mandakovic et al. 2018). However, using eDNA for these

718 tests remains only possible at a coarse level of classification because reference sequence

719 databases are incomplete and metabarcodes are not completely diagnostic. Ongoing efforts to

720 sequence species and build a global taxonomic biodiversity database in the next decade (e.g. the

721 Earth BioGenome Project, Lewin et al. 2018; the Centre for Biodiversity Genomics, Hobern

722 2020) are positioned to ameliorate this challenge in the coming years. 
724 confidence and resolution of eDNA-based detection is crucial for effective biomonitoring. First,

725 a detection bias in favor of small body size is evident in eDNA studies (Figure 1; Tables S5,S6).

726 Including other loci in metabarcoding (e.g. Andersen et al. 2012) or employing DNA capture

727 approaches to target larger organisms (Seeber et al. 2019) may improve detection of large-bodied

728 species. Novel tools such as camera traps or satellite telemetry (e.g. NASA CubeSats) to increase

729 detectability for large organisms could be used to complement eDNA results, as could more

730 participation by CCS to add iNaturalist data and to scrutinize eDNA results (as helped produce

731 the TOS score; Table S6). Second, different DNA extractions from the same sample exhibit

732 taxonomic heterogeneity (Text S3; Table S4). We are devoting efforts to examining stability and

733 stochasticity of taxonomic profiles under varied sample processing (Castro et al. in prep) and

734 DNA library preparation steps (Shirazi et al. in prep) in response to calls for attention to these

735 potential biases (Prosser 2010, Goldberg et al. 2016), and were careful in this study to use

736 approaches that are considered a standard of the field for reducing these biases.

738 can advance biodiversity assessment capacity. Space, flight, tower and drone-based remote

739 sensing information is becoming increasingly available and accessible (Pettorelli et al. 2014).

740 These data now can provide more direct, spatially continuous measures of plant functional

741 diversity and ecosystem functioning at regional (Schneider et al. 2017, Durán et al. 2019) to

742 global scales (Schimel et al. 2019, Bae et al. 2019). Using remotely sensed variables will not

743 only elucidate classical community-environment interactions, but can also provide an

744 opportunity to predict biodiversity turnover across large landscapes even when biological

745 samples from all locations are difficult to acquire (Bush et al. 2017). 
In conclusion, we demonstrate the emerging potential of coupling CCS observations and

747 eDNA data from samples that CCS volunteers collect with remote sensing and ecological

748 modeling to assess community-environment interactions and ultimately map community

749 turnover. We provide one of the most comprehensive surveys of terrestrial biodiversity across

750 three domains of life over a large, environmentally diverse state. We show the predictive and

751 explanatory power of environmental variables on alpha, beta, and zeta diversity across highly

752 diverse regions and at local geographic scales. The beta diversity map for California, illustrating

753 a continuous surface of community turnover, is validated by its similarity to the standard US

754 Ecoregion maps. Computationally intensive and artificial intelligence driven models are

755 producing maps for mitigating the challenges of global change (Stephenson et al. 2018,

756 Harfouche et al. 2019). Our approach contributes to the development of strategies to model

757 living systems by integrating molecular-assisted observations with traditional and remotely

758 sensed biodiversity measures. By encouraging more eDNA sequencing and CCS across broad

759 regions in narrow temporal windows, and by systematically using Essential Biodiversity

760 Variables in analyses, a new arsenal can be applied to tracking and predicting ecological change.

761 Such data will allow the dissection of the contributions of biotic and abiotic factors to system

762 resilience. Multidisciplinary initiatives that include specialists and CCS together can energize a

763 necessary constituency for conservation.

\section{Data availability}

765 Scripts and data associated with the analyses are archived in Zenodo archive (link

766 pending) and github repository (https://github.com/meixilin/caledna transect). The raw

767 sequencing data will be deposited in the NCBI Sequence Reads Archive (accession pending). 


\section{$\underline{\text { Author Contributions }}$}

RSM, RKW, BAS, EEC designed the study. ML, RSM, RH, ALS, MO, EEC and ZG

770 selected analyses. RSM and EEC coordinated public sampling. ML, EF, TAS, and RSM made

771 DNA libraries. ML, MPM, FS, AGV, DRR, RSM and EEC curated environmental metadata. ML,

772 FS, and RH generated statewide data layers. ML led biodiversity and gradient forest analyses,

773 and ML, ALS, and RSM generated plots. EJM generated the synthetic phylogeny. All authors

774 performed analyses and interpretation. ML, RSM, and RKW wrote the manuscript with input

775 from all authors.

\section{Acknowledgement}

Funding for the CALeDNA sample processing, infrastructure, and personnel was

778 provided by the University of California Research Initiatives (UCRI) Catalyst grant CA-16-

779376437 and Howard Hughes Medical Institute (HHMI) Professors Grant GT10483. Additional

780 funding for personnel and computational infrastructure was provided by the National Science

781 Foundation (NSF) 1759756. The research carried out at the Jet Propulsion Laboratory, California

782 Institute of Technology, was under a contract with the National Aeronautics and Space

783 Administration (80NM0018D0004). Government sponsorship is acknowledged. Graduate

784 student support was additionally provided by the National Council for Scientific and

785 Technological Development of Brazil [Grant No. 209261/2014-5] and the University of

786 California, Los Angeles Department of Ecology and Evolutionary Biology (EEB) Summer

787 Research Fellowship. We thank the UC Natural Reserves System managers, other natural areas

788 managers, and the hundreds of volunteers for collections. We thank A. Mahinan, N. Stavros, W.-

789 Y Kwan, for assisting with spatial environmental data and to A. DeVries and L. Bulbenko for

790 preparing extractions. We thank C.M. Mueller and Z. Kurtz for help optimizing analyses. 


\section{Literature cited}

792 Amaral-Zettler, L. A., E. A. McCliment, H. W. Ducklow, and S. M. Huse. 2009. A Method for Studying Protistan Diversity Using Massively Parallel Sequencing of V9 Hypervariable Regions of Small-Subunit Ribosomal RNA Genes. PLOS ONE 4:e6372.

Andersen, K., K. L. Bird, M. Rasmussen, J. Haile, H. Breuning-Madsen, et al. 2012. Meta-

Bae, S., S. R. Levick, L. Heidrich, P. Magdon, B. F. Leutner, et al. 2019. Radar vision in the mapping of forest biodiversity from space. Nature Communications 10:4757.

Bahram, M., F. Hildebrand, S. K. Forslund, J. L. Anderson, N. A. Soudzilovskaia, et al. 2018. Structure and function of the global topsoil microbiome. Nature 560:233-237. Species richness and endemism in the native flora of California. American Journal of Botany 104:487-501.

Bay, R. A., R. J. Harrigan, V. L. Underwood, H. L. Gibbs, T. B. Smith, and K. Ruegg. 2018. Genomic signals of selection predict climate-driven population declines in a migratory bird. Science 359:83-86. DNA for wildlife biology and biodiversity monitoring. Trends in Ecology \& Evolution 
814 Bolger, D. T., A. V. Suarez, K. R. Crooks, S. A. Morrison, and T. J. Case. 2000. Arthropods in 815 urban habitat fragments in southern California: area, age, and edge effects. Ecological $816 \quad$ Applications 10:1230-1248.

817 Breed, M. F., P. A. Harrison, C. Blyth, M. Byrne, V. Gaget, et al. 2019. The potential of genomics for restoring ecosystems and biodiversity. Nature Reviews Genetics 20:615-

Breiman, L. 2001. Statistical Modeling: The Two Cultures (with comments and a rejoinder by the author). Statistical Science 16:199-231.

Bush, A., R. Sollmann, A. Wilting, K. Bohmann, B. Cole, et al. 2017. Connecting Earth observation to high-throughput biodiversity data. Nature Ecology \& Evolution 1:0176.

824 Callahan, B. J., P. J. McMurdie, M. J. Rosen, A. W. Han, A. J. A. Johnson, and S. P. Holmes. 2016. DADA2: High-resolution sample inference from Illumina amplicon data. Nature Methods 13:581-583.

Caporaso, J. G., C. L. Lauber, W. A. Walters, D. Berg-Lyons, J. Huntley, et al. 2012. Ultra-highthroughput microbial community analysis on the Illumina HiSeq and MiSeq platforms. The ISME Journal 6:1621-1624.

833 Ceballos, G., P. R. Ehrlich, A. D. Barnosky, A. Garcia, R. M. Pringle, and T. M. Palmer. 2015. Accelerated modern human-induced species losses: Entering the sixth mass extinction. Science Advances 1:e1400253-e1400253. 
836 Cheng, J., B. Karambelkar, and Y. Xie. 2019. leaflet: Create Interactive Web Maps with the JavaScript "Leaflet" Library.

838 Collins, C. G., J. E. Stajich, S. E. Weber, N. Pombubpa, and J. M. Diez. 2018. Shrub range expansion alters diversity and distribution of soil fungal communities across an alpine

841 Cristescu, M. E. 2014. From barcoding single individuals to metabarcoding biological communities: towards an integrative approach to the study of global biodiversity. Trends in Ecology \& Evolution 29:566-571.

844 Crowther, T. W., J. van den Hoogen, J. Wan, M. A. Mayes, A. D. Keiser, et al. 2019. The global soil community and its influence on biogeochemistry. Science 365:eaav0550.

846 Curd, E. E., Z. Gold, G. S. Kandlikar, J. Gomer, M. Ogden, et al. 2019. Anacapa Toolkit : an environmental DNA toolkit for processing multilocus metabarcode datasets. Methods in Ecology and Evolution:2041-210X.13214.

849 Deiner, K., H. M. Bik, E. Mächler, M. Seymour, A. Lacoursière-Roussel, et al. 2017. Environmental DNA metabarcoding: Transforming how we survey animal and plant DNA reveals that rivers are conveyer belts of biodiversity information. Nature Communications 7:12544. 
Demarais, S., J. P. Verschuyl, G. J. Roloff, D. A. Miller, and T. B. Wigley. 2017. Tamm review: terrestrial vertebrate biodiversity and intensive forest management in the US. Forest Ecology and Management 385:308-330.

Díaz, S., J. Settele, E. S. Brondízio, H. T. Ngo, J. Agard, et al. 2019. Pervasive human-driven decline of life on Earth points to the need for transformative change. Science 366:eaax3100.

Durán, S. M., R. E. Martin, S. Díaz, B. S. Maitner, Y. Malhi, et al. 2019. Informing trait-based ecology by assessing remotely sensed functional diversity across a broad tropical

Ehrlich, R., S. Schulz, M. Schloter, and Y. Steinberger. 2015. Effect of slope orientation on microbial community composition in different particle size fractions from soils obtained from desert ecosystems. Biology and Fertility of Soils 51:507-510.

Ellis, N., S. J. Smith, and C. R. Pitcher. 2012. Gradient forests: calculating importance gradients on physical predictors. Ecology 93:156-168. environmental metabarcodes for analysing soil DNA: potential for studying past and present ecosystems. Molecular Ecology 21:1821-1833. 
Erlandson, S., X. Wei, J. Savage, J. Cavender-Bares, and K. Peay. 2018. Soil abiotic variables are more important than Salicaceae phylogeny or habitat specialization in determining soil microbial community structure. Molecular Ecology 27:2007-2024.

Ferrier, S., and A. Guisan. 2006. Spatial modelling of biodiversity at the community level. Journal of Applied Ecology 43:393-404.

Fick, S. E., and R. J. Hijmans. 2017. WorldClim 2: new 1-km spatial resolution climate surfaces for global land areas. International Journal of Climatology 37:4302-4315.

Fierer, N., and R. B. Jackson. 2006. The diversity and biogeography of soil bacterial communities. Proceedings of the National Academy of Sciences 103:626-631.

Fitzpatrick, M. C., and S. R. Keller. 2015. Ecological genomics meets community-level

Franklin, T. W., K. S. McKelvey, J. D. Golding, D. H. Mason, J. C. Dysthe, et al. 2019. Using environmental DNA methods to improve winter surveys for rare carnivores: DNA from snow and improved noninvasive techniques. Biological Conservation 229:50-58.

894 Gao, X., H. Lin, K. Revanna, and Q. Dong. 2017. A Bayesian taxonomic classification method for 16S rRNA gene sequences with improved species-level accuracy. BMC dryland legumes in Baja California, Mexico. Journal of Vegetation Science 14:475-486. scale trends of microbial and animal biodiversity revealed across diverse temperate soil ecosystems. Nature Communications 10:1107. 
902

903

904

905

906

907

908

909

910

911

912

913

914

915

916

917

918

919

920

921

922

923

924

Gibson, N., R. Meissner, A. S. Markey, and W. A. Thompson. 2012. Patterns of plant diversity in ironstone ranges in arid south western Australia. Journal of Arid Environments 77:2531.

Goldberg, C. S., C. R. Turner, K. Deiner, K. E. Klymus, P. F. Thomsen, et al. 2016. Critical considerations for the application of environmental DNA methods to detect aquatic species. Methods in Ecology and Evolution:1299-1307.

Gordon, A., G. Hannon, and others. 2010. Fastx-toolkit. FASTQ/A short-reads preprocessing tools (unpublished) http://hannonlab. cshl. edu/fastx_toolkit 5.

Groenendyk, D. G., T. P. A. Ferré, K. R. Thorp, and A. K. Rice. 2015. Hydrologic-ProcessBased Soil Texture Classifications for Improved Visualization of Landscape Function. PLOS ONE 10:e0131299.

Gu, W., J. Song, Y. Cao, Q. Sun, H. Yao, et al. 2013. Application of the ITS2 Region for Barcoding Medicinal Plants of Selaginellaceae in Pteridophyta. PLOS ONE 8:e67818.

Harfouche, A. L., D. A. Jacobson, D. Kainer, J. C. Romero, A. H. Harfouche, et al. 2019. Accelerating Climate Resilient Plant Breeding by Applying Next-Generation Artificial Intelligence. Trends in Biotechnology 37:1217-1235.

Hennessy, S. M., S. A. Marczak, L. A. Nordstrom, and R. R. Swaisgood. 2018. Reconsidering habitat associations in the Anthropocene. Global Ecology and Conservation 14:e00397.

Hijmans, R. J. 2017. raster: Geographic Data Analysis and Modeling.

Hobern, D. G. 2020. BIOSCAN: DNA Barcoding to accelerate taxonomy and biogeography for conservation and sustainability. Genome.

van den Hoogen, J., S. Geisen, D. Routh, H. Ferris, W. Traunspurger, et al. 2019. Soil nematode abundance and functional group composition at a global scale. Nature 572:194-198. 
925 Hopper, S. D. 1979. Biogeographical aspects of speciation in the southwest Australian flora. Annual review of ecology and systematics 10:399-422.

Horner-Devine, M. C., J. M. Silver, M. A. Leibold, B. J. M. Bohannan, R. K. Colwell, et al. 2007. A Comparison of Taxon Co-Occurrence Patterns for Macro- and Microorganisms.

Hui, C., M. A. McGeoch, A. E. S. Harrison, and E. J. L. Bronstein. 2014. Zeta Diversity as a Concept and Metric That Unifies Incidence-Based Biodiversity Patterns. The American Naturalist 184:684-694.

Hui, C., W. Vermeulen, and G. Durrheim. 2018. Quantifying multiple-site compositional turnover in an Afrotemperate forest, using zeta diversity. Forest Ecosystems 5:15.

935 Jackson, L. E., T. M. Bowles, H. Ferris, A. J. Margenot, A. Hollander, et al. 2019. Plant and soil microfaunal biodiversity across the borders between arable and forest ecosystems in a

938 Jenkins, C. N., S. L. Pimm, and L. N. Joppa. 2013. Global patterns of terrestrial vertebrate 939 diversity and conservation. Proceedings of the National Academy of Sciences 110:E2602-E2610.

941 Jenkins, C. N., K. S. Van Houtan, S. L. Pimm, and J. O. Sexton. 2015. US protected lands 942 mismatch biodiversity priorities. Proceedings of the National Academy of Sciences 112:5081-5086.

944 Jetz, W., M. A. McGeoch, R. Guralnick, S. Ferrier, J. Beck, et al. 2019. Essential biodiversity

945 variables for mapping and monitoring species populations. Nature Ecology \&

$946 \quad$ Evolution:1. 
947 Kadir, T., L. Mazur, C. Milanes, and K. Randles. 2013. Indicators of climate change in

948 California. Office of Environmental Health Hazard Assessment, California Environmental Protection Agency, Sacramento, California.

950 Kandlikar, G. S., Z. J. Gold, M. C. Cowen, R. S. Meyer, A. C. Freise, et al. 2018. ranacapa: An R package and Shiny web app to explore environmental DNA data with exploratory statistics and interactive visualizations. F1000Research 7:1734.

953 Karimi, B., S. Terrat, S. Dequiedt, N. P. A. Saby, W. Horrigue, et al. 2018. Biogeography of soil bacteria and archaea across France. Science Advances 4:eaat1808.

955 Kerr, J. T. 1999. Weak links: 'Rapoport's rule' and large-scale species richness patterns. Global Ecology and Biogeography 8:47-54.

957 Kobori, H., J. L. Dickinson, I. Washitani, R. Sakurai, T. Amano, et al. 2016. Citizen science: a new approach to advance ecology, education, and conservation. Ecological Research $31: 1-19$.

960 Konopka, A. 2009. What is microbial community ecology? The ISME Journal 3:1223-1230.

961 Koskella, B., L. J. Hall, and C. J. E. Metcalf. 2017. The microbiome beyond the horizon of 962 ecological and evolutionary theory. Nature Ecology \& Evolution 1:1606.

963 Kurtz, Z. D., C. L. Müller, E. R. Miraldi, D. R. Littman, M. J. Blaser, and R. A. Bonneau. 2015. 964 Sparse and Compositionally Robust Inference of Microbial Ecological Networks. PLOS $965 \quad$ Computational Biology 11:e1004226.

966 Lallias, D., J. G. Hiddink, V. G. Fonseca, J. M. Gaspar, W. Sung, et al. 2015. Environmental 967 metabarcoding reveals heterogeneous drivers of microbial eukaryote diversity in contrasting estuarine ecosystems. The ISME Journal 9:1208-1221. 
969 Langmead, B., and S. L. Salzberg. 2012. Fast gapped-read alignment with Bowtie 2. Nature Methods 9:357-359.

971 Lanzén, A., K. Lekang, I. Jonassen, E. M. Thompson, and C. Troedsson. 2017. DNA extraction replicates improve diversity and compositional dissimilarity in metabarcoding of eukaryotes in marine sediments. PLOS ONE 12:e0179443.

974 Latombe, G., M. A. McGeoch, D. A. Nipperess, and C. Hui. 2018. zetadiv: Functions to Compute Compositional Turnover Using Zeta Diversity.

976 Lejzerowicz, F., P. Esling, L. Pillet, T. A. Wilding, K. D. Black, and J. Pawlowski. 2015. Highthroughput sequencing and morphology perform equally well for benthic monitoring of

979 Leray, M., J. Y. Yang, C. P. Meyer, S. C. Mills, N. Agudelo, et al. 2013. A new versatile primer marine ecosystems. Scientific Reports 5:1-10.

983 Lewin, H. A., G. E. Robinson, W. J. Kress, W. J. Baker, J. Coddington, et al. 2018. Earth

984 BioGenome Project: Sequencing life for the future of life. Proceedings of the National Academy of Sciences:201720115.

986 Li, X., H. Zhu, S. Geisen, C. Bellard, F. Hu, et al. 2020. Agriculture erases climate constraints on soil nematode communities across large spatial scales. Global Change Biology 26:919_ 930. 
990

991

992

993

994

995

996

997

998

999

1000

1001

1002

1003

1004

1005

1006

1007

1008

1009

1010

1011

1012

Mandakovic, D., C. Rojas, J. Maldonado, M. Latorre, D. Travisany, et al. 2018. Structure and cooccurrence patterns in microbial communities under acute environmental stress reveal ecological factors fostering resilience. Scientific Reports 8:1-12.

Martin, M. 2011. Cutadapt removes adapter sequences from high-throughput sequencing reads. EMBnet. journal 17:10-12.

McKnight, M. W., P. S. White, R. I. McDonald, J. F. Lamoreux, W. Sechrest, et al. 2007. Putting Beta-Diversity on the Map: Broad-Scale Congruence and Coincidence in the Extremes. PLOS Biology 5:e272.

Mevik, B.-H., R. Wehrens, and K. H. Liland. 2019. pls: Partial Least Squares and Principal Component Regression.

Meyer, R., E. E. Curd, T. Schweizer, Z. Gold, D. R. Ruiz, et al. 2019. The California Environmental DNA “CALeDNA” Program. California Agriculture Accepted.

Miralles, L., E. Dopico, F. Devlo-Delva, and E. Garcia-Vazquez. 2016. Controlling populations of invasive pygmy mussel (Xenostrobus securis) through citizen science and environmental DNA. Marine Pollution Bulletin 110:127-132.

Miya, M., Y. Sato, T. Fukunaga, T. Sado, J. Y. Poulsen, et al. 2015. MiFish, a set of universal PCR primers for metabarcoding environmental DNA from fishes: detection of more than 230 subtropical marine species. Open Science 2:150088.

Montagna, M., A. Berruti, V. Bianciotto, P. Cremonesi, R. Giannico, et al. 2018. Differential biodiversity responses between kingdoms (plants, fungi, bacteria and metazoa) along an Alpine succession gradient. Molecular Ecology 27:3671-3685.

Myers, N., R. A. Mittermeier, C. G. Mittermeier, G. A. B. da Fonseca, and J. Kent. 2000. Biodiversity hotspots for conservation priorities. Nature 403:853-858. 
1013 Ogle, D. H., P. Wheeler, and A. Dinno. 2019. FSA: Fisheries Stock Analysis.

1014 Oksanen, J., F. G. Blanchet, M. Friendly, R. Kindt, P. Legendre, et al. 2019. vegan: Community

1015 Ecology Package.

1016 Omernik, J. M., and G. E. Griffith. 2014. Ecoregions of the Conterminous United States: Evolution of a Hierarchical Spatial Framework. Environmental Management 54:1249_

$1018 \quad 1266$.

1019 Parducci, L., K. D. Bennett, G. F. Ficetola, I. G. Alsos, Y. Suyama, et al. 2017. Ancient plant 1020 DNA in lake sediments. New Phytologist 214:924-942.

1021 Pearce-Higgins, J. W., S. R. Baillie, K. Boughey, N. A. D. Bourn, R. P. B. Foppen, et al. 2018.

1022 Overcoming the challenges of public data archiving for citizen science biodiversity

1023 recording and monitoring schemes. Journal of Applied Ecology 55:2544-2551.

1024 Pereira, H. M., S. Ferrier, M. Walters, G. N. Geller, R. H. G. Jongman, et al. 2013. Essential 1025 Biodiversity Variables. Science 339:277-278.

1026 Peters, M. K., A. Hemp, T. Appelhans, J. N. Becker, C. Behler, et al. 2019. Climate-land-use interactions shape tropical mountain biodiversity and ecosystem functions. Nature:1.

1028 Pettorelli, N., K. Safi, and W. Turner. 2014. Satellite remote sensing, biodiversity research and conservation of the future. Phil. Trans. R. Soc. B 369:20130190.

1030 Pimm, S. L., C. N. Jenkins, R. Abell, T. M. Brooks, J. L. Gittleman, et al. 2014. The biodiversity of species and their rates of extinction, distribution, and protection. Science 344:1246752.

1032 Pitcher, C. R., N. Ellis, and S. J. Smith. 2011. Example analysis of biodiversity survey data with R package gradientForest:16. 
1034 Pitcher, C. R., P. Lawton, N. Ellis, S. J. Smith, L. S. Incze, et al. 2012. Exploring the role of 1035 environmental variables in shaping patterns of seabed biodiversity composition in 1036 regional-scale ecosystems. The Journal of Applied Ecology 49:670-679.

1037 Prober, S. M., J. W. Leff, S. T. Bates, E. T. Borer, J. Firn, et al. 2015. Plant diversity predicts 1038 beta but not alpha diversity of soil microbes across grasslands worldwide. Ecology $1039 \quad$ Letters 18:85-95.

1040 Prosser, J. I. 2010. Replicate or lie. Environmental Microbiology 12:1806-1810.

1041 Prosser, J. I. 2015. Dispersing misconceptions and identifying opportunities for the use of "omics" 1042 in soil microbial ecology. Nature Reviews Microbiology 13:439-446.

1043 Pryke, J. S., and M. J. Samways. 2009. Conservation of the insect assemblages of the Cape

1044 Peninsula biodiversity hotspot. Journal of Insect Conservation 13:627.

1045 R Core Team. 2019. R: A Language and Environment for Statistical Computing. R Foundation 1046 for Statistical Computing, Vienna, Austria.

1047 Ranjard, L., S. Dequiedt, N. Chemidlin Prévost-Bouré, J. Thioulouse, N. P. A. Saby, et al. 2013.

1048 Turnover of soil bacterial diversity driven by wide-scale environmental heterogeneity.

$1049 \quad$ Nature Communications 4:1-10.

1050 Schimel, D., F. D. Schneider, and JPL Carbon and Ecosystem Participants. 2019. Flux towers in 1051 the sky: global ecology from space. New Phytologist 224:570-584.

1052 Schneider, F. D., F. Morsdorf, B. Schmid, O. L. Petchey, A. Hueni, et al. 2017. Mapping 1053 functional diversity from remotely sensed morphological and physiological forest traits. $1054 \quad$ Nature Communications 8:1441.

1055 Sebastià, M.-T. 2007. Plant guilds drive biomass response to global warming and water 1056 availability in subalpine grassland. Journal of Applied Ecology 44:158-167. 
1057 Seeber, P. A., G. K. McEwen, U. Löber, D. W. Förster, M. L. East, et al. 2019. Terrestrial mammal surveillance using hybridization capture of environmental DNA from African waterholes. Molecular Ecology Resources 19:1486-1496.

Sessitsch, A., A. Weilharter, M. H. Gerzabek, H. Kirchmann, and E. Kandeler. 2001. Microbial Population Structures in Soil Particle Size Fractions of a Long-Term Fertilizer Field environment for integrated models of biomolecular interaction networks. Genome research 13:2498-2504.

Shirazi, S., R. S. Meyer, and B. Shapiro. in prep. How many PCR replicates are enough in environmental DNA metabarcoding? In prep.

Silva, A. C., and A. F. Souza. 2018. Aridity drives plant biogeographical sub regions in the

Simons, A. L., R. Mazor, E. D. Stein, and S. Nuzhdin. 2019. Using alpha, beta, and zeta diversity Caatinga, the largest tropical dry forest and woodland block in South America. PLOS ONE 13:e0196130.

1074 Ssekagiri, A., W. Sloan, and U. Ijaz. 2017. microbiomeSeq: An R package for analysis of microbial communities in an environmental context.

1076 Stephenson, F., J. R. Leathwick, S. W. Geange, R. H. Bulmer, J. E. Hewitt, et al. 2018. Using 1077 Gradient Forests to summarize patterns in species turnover across large spatial scales and 1078 inform conservation planning. Diversity and Distributions 24:1641-1656. 
Stevens, G. C. 1989. The latitudinal gradient in geographical range: how so many species coexist in the tropics. The American Naturalist 133:240-256.

1081 Sutter, M., and A. Kinziger. 2018. Develop a Tidewater Goby Survey Method Using Environmental DNA.

1083

1084

1085

1086

1087

1088

1089

1090

1091

1092

1093

1094

1095

1096

1097

1098

1099

1100

Taberlet, P., A. Bonin, L. Zinger, and E. Coissac. 2018. Environmental DNA: For Biodiversity Research and Monitoring. Oxford University Press.

Tedersoo, L., M. Bahram, S. Põlme, U. Kõljalg, N. S. Yorou, et al. 2014. Global diversity and geography of soil fungi. Science 346:1256688.

Theobald, E. J., A. K. Ettinger, H. K. Burgess, L. B. DeBey, N. R. Schmidt, et al. 2015. Global change and local solutions: Tapping the unrealized potential of citizen science for biodiversity research. Biological Conservation 181:236-244.

Thomas, A. C., P. L. Nguyen, J. Howard, and C. S. Goldberg. 2019. A self-preserving, partially biodegradable eDNA filter. Methods in Ecology and Evolution 10:1136-1141.

Thompson, L. R., J. G. Sanders, D. McDonald, A. Amir, J. Ladau, et al. 2017. A communal catalogue reveals Earth's multiscale microbial diversity. Nature 551:457.

Timling, I., D. A. Walker, C. Nusbaum, N. J. Lennon, and D. L. Taylor. 2014. Rich and cold: diversity, distribution and drivers of fungal communities in patterned-ground ecosystems of the North American Arctic. Molecular Ecology 23:3258-3272.

Tipton, L., C. L. Müller, Z. D. Kurtz, L. Huang, E. Kleerup, et al. 2018. Fungi stabilize connectivity in the lung and skin microbial ecosystems. Microbiome 6:12.

U.S. Environmental Protection Agency. 2010, May 1. NA_CEC_Eco_Level2. U.S. EPA Office of Research and Development (ORD) - National Health and Environmental Effects 
Research Laboratory (NHEERL), Corvallis, OR. $\mathrm{ftp}: / / \mathrm{ftp}$. epa.gov/wed/ecoregions/cec_na/NA_CEC_Eco_Level2.zip ORD - NHEERL, Corvallis, OR. ftp://newftp.epa.gov/EPADataCommons/ORD/Ecoregions/ca/ca_eco_13.zip

USDA Forest Service. 2007. USDA Ecoregion Sections, California. USDA Forest Service Pacific Southwest Region - Remote Sensing Lab. https://databasin.org/datasets/81a3a809a2ae4c099f2e495c0b2ecc91

1109 Větrovský, T., P. Kohout, M. Kopecký, A. Machac, M. Man, et al. 2019. A meta-analysis of global fungal distribution reveals climate-driven patterns. Nature Communications 10:1-

Waller, J. 2019, January 21. Will citizen science take over? https://data-blog.gbif.org/post/gbifcitizen-science-data/.

1114 Wang, R., J. A. Gamon, J. Cavender-Bares, P. A. Townsend, and A. I. Zygielbaum. 2018. The spatial sensitivity of the spectral diversity-biodiversity relationship: an experimental test

1117 Warton, D. I., F. G. Blanchet, R. B. O’Hara, O. Ovaskainen, S. Taskinen, et al. 2015. So Many

1118 Variables: Joint Modeling in Community Ecology. Trends in Ecology \& Evolution $1119 \quad 30: 766-779$.

1120 Wildlife Conservation Society and Center for International Earth Science Information Network, 1121 Columbia University. 2005. Last of the Wild Project, Version 2, 2005 (LWP-2): Global 1122 Human Footprint Dataset (Geographic). Palisades, NY: NASA Socioeconomic Data and 1123 Applications Center (SEDAC). https://doi.org/10.7927/H4M61H5F. 
1124 Weier, J., and D. Herring. 2000, August 30. Measuring Vegetation (NDVI \& EVI).

1125 https:/earthobservatory.nasa.gov/features/MeasuringVegetation.

1126 White, T. J., T. Bruns, S. Lee, J. Taylor, and others. 1990. Amplification and direct sequencing 1127 of fungal ribosomal RNA genes for phylogenetics. PCR protocols: a guide to methods $1128 \quad$ and applications 18:315-322.

1129 Yamasaki, E., F. Altermatt, J. Cavender-Bares, M. C. Schuman, D. Zuppinger-Dingley, et al.

1130 2017. Genomics meets remote sensing in global change studies: monitoring and

1131 predicting phenology, evolution and biodiversity. Current Opinion in Environmental

$1132 \quad$ Sustainability 29:177-186.

1133 Yang, T., J. M. Adams, Y. Shi, J. He, X. Jing, et al. 2017. Soil fungal diversity in natural 1134 grasslands of the Tibetan Plateau: associations with plant diversity and productivity. New $1135 \quad$ Phytologist 215:756-765.

1136 Yu, D. W., Y. Ji, B. C. Emerson, X. Wang, C. Ye, et al. 2012. Biodiversity soup: metabarcoding 1137 of arthropods for rapid biodiversity assessment and biomonitoring: Biodiversity soup. $1138 \quad$ Methods in Ecology and Evolution 3:613-623.

1139 Zarnetske, P. L., Q. D. Read, S. Record, K. D. Gaddis, S. Pau, et al. 2019. Towards connecting 1140 biodiversity and geodiversity across scales with satellite remote sensing. Global Ecology $1141 \quad$ and Biogeography 28:548-556. 


\section{Table 1}

1145 Table 1 List of the categorical and a reduced set of numerical variables used in the diversity analysis and gradient forest modeling. For

1146 a complete list of variables, detailed description and data URL, refer to Table S1.

\begin{tabular}{lll}
\hline Variable & Category & Description and definition \\
\hline $\begin{array}{ll}\text { Categorical } \\
\text { loc }\end{array}$ & Lociables & \\
clust & Location & Name of places visited reported by volunteers \\
ecoregion & Habitat & Neighboring cluster of sites within a radius of 0.5 km derived from GPS record \\
majorhab & Habitat & MPA Level III Ecoregions of California (Conterminous United States) \\
minorhab & Habitat & Minor habitat type classified according to California Wildlife Habitat Relationships System \\
transect & Habitat & Original classification of the predominant biome type (coast/coastal, shrub/ShrubScrub, and forest) \\
NLCD & Habitat & USGS national land cover classification 2011 \\
SoS & Soil Properties & Volunteers' classification of substrate type (Sediment, Soil, Sand) \\
taxousda & Soil Properties & Predicted most probable class in USDA soil taxonomy \\
Reduced set & of numerical variables \\
Longitude & Location & Longitude of sample sites \\
hfp & Habitat & Global human footprint index \\
bio1 & BioClim & Annual Mean Temperature \\
bio2 & BioClim & Mean Diurnal Range (Mean of monthly (max temp - min temp)) \\
bio3 & BioClim & Isothermality (BIO2/BIO7) (*100) \\
bio4 & BioClim & Temperature Seasonality (standard deviation *100) \\
bio5 & BioClim & Max Temperature of Warmest Month \\
bio6 & BioClim & Min Temperature of Coldest Month \\
bio8 & BioClim & Mean Temperature of Wettest Quarter \\
bio14 & BioClim & Precipitation of Driest Month \\
bio15 & BioClim & Precipitation Seasonality (Coefficient of Variation) \\
phihox & Soil Properties & Soil pH x 10 in H2O at depth 0.00 m \\
& &
\end{tabular}




\begin{tabular}{lll}
\hline orcdrc & Soil Properties & Soil organic carbon content (fine earth fraction) in g per kg at depth 0.00 m \\
cecsol & Soil Properties & Cation exchange capacity of soil in cmolc/kg at depth $0.00 \mathrm{~m}$ \\
sndppt & Soil Properties & Sand content 50 to $2000 \mu \mathrm{m})$ mass fraction in \% at depth $0.00 \mathrm{~m}$ \\
bldfie & Soil Properties & Bulk density (fine earth) in $\mathrm{kg} /$ cubic meter at depth $0.00 \mathrm{~m}$ \\
ntot & Soil Properties & Weight percentage of total nitrogen \\
elev & Topography & Elevation of sampling site \\
Slope & Topography & The rate of change of elevation for each digital elevation model (DEM) cell \\
aspect & Topography & The direction of the maximum rate of change in the z-value from each cell in a raster surface \\
CTI & Topography & Compound Topographic Index \\
DAH & Topography & Diurnal Anisotropic Heating \\
B1 & Vegetation & Sentinel-2 spectral band 1 (Wavelength: 443.9nm (S2A) / 442.3nm (S2B); Description: Aerosols) \\
B4 & Vegetation & Sentinel-2 spectral band 4 (Wavelength: 664.5nm (S2A) / 665nm (S2B); Description: Red) \\
B6 & Vegetation & Sentinel-2 spectral band 6 (Wavelength: 740.2nm (S2A) / 739.1nm (S2B); Description: Red Edge 2) \\
B9 & Vegetation & Sentinel-2 spectral band 9 (Wavelength: 945nm (S2A)/ 943.2nm (S2B); Description: Water vapor) \\
B10 & Vegetation & Sentinel-2 spectral band 10 (Wavelength: $1373.5 \mathrm{~nm}(\mathrm{~S} 2 \mathrm{~A}) / 1376.9 \mathrm{~nm}$ (S2B); Description: Cirrus) \\
B11 & Vegetation & Sentinel-2 spectral band 11 (Wavelength: $1613.7 \mathrm{~nm}(\mathrm{~S} 2 \mathrm{~A}) / 1610.4 \mathrm{~nm}$ (S2B); Description: SWIR 1) \\
NDVI32 & Vegetation & Normalized Difference Vegetation Index in 32 days period \\
NBRT & Vegetation & Normalized Burn Ratio Thermal (NBRT) index in 32 days period \\
greenness & Vegetation & Annual Greenest Pixel in the year of 2017 \\
imprv & Habitat & Percent of the pixel covered by developed impervious surface \\
ptrcv & Habitat & Percent of the pixel that's covered by tree canopy \\
\hline
\end{tabular}




\section{Table 2}

1149 Table 2 Envfit result on PCoA ordination for each metabarcode. Here we present the three

1150 significant $(\mathrm{P}<0.001)$ environmental variables with the highest correlation coefficient. The

1151 significance of the correlation was tested by 1999 permutations. For a complete result of all

1152 variables, please refer to Table S12. The direction of changes is included in Figure S20.

\begin{tabular}{lllllll}
\hline Metabarcode & 1st variable & $\mathbf{R}^{\mathbf{2}}$ & 2nd variable & $\mathbf{R}^{\mathbf{2}}$ & 3rd variable & $\mathbf{R}^{\mathbf{2}}$ \\
\hline 16S & NDVI32 & 0.49 & greenness & 0.47 & $\mathrm{~B} 1$ & 0.42 \\
18S & NDVI32 & 0.51 & greenness & 0.49 & $\mathrm{~B} 1$ & 0.43 \\
CO1 & orcdrc & 0.41 & ptrcv & 0.36 & NBRT & 0.33 \\
FITS & greenness & 0.52 & B1 & 0.5 & orcdrc & 0.46 \\
PITS & bio3 & 0.21 & sndppt & 0.2 & B11 & 0.13 \\
\hline
\end{tabular}

1153 


\section{Table 3}

1155 Table 3 Variation in $\zeta_{4}$ diversity, for communities defined at the family level, attributed to

1156 geographic separation distance between samples versus variation in an environmental factor

1157 group for those same samples. Within each metabarcode, factor groups were ordered from lowest

1158 to highest contributions to variations in zeta diversity.

\begin{tabular}{llllll}
\hline Metabarcode & FactorGroup & NumSamples & VarFactor & VarDistance & VarUnknown \\
\hline $16 S$ & Location & 184 & $0.00 \%$ & $0.29 \%$ & $99.70 \%$ \\
$16 S$ & Topography & 184 & $0.94 \%$ & $0.17 \%$ & $98.90 \%$ \\
$16 S$ & Habitat & 156 & $1.33 \%$ & $0.00 \%$ & $98.70 \%$ \\
$16 S$ & Vegetation & 169 & $5.92 \%$ & $0.00 \%$ & $94.10 \%$ \\
$16 S$ & BioClim & 184 & $7.17 \%$ & $0.00 \%$ & $92.20 \%$ \\
$16 S$ & Soil Properties & 180 & $9.21 \%$ & $0.00 \%$ & $90.70 \%$ \\
$18 S$ & Location & 184 & $0.14 \%$ & $0.00 \%$ & $99.90 \%$ \\
$18 S$ & Habitat & 156 & $5.49 \%$ & $0.00 \%$ & $94.50 \%$ \\
$18 S$ & Topography & 184 & $7.15 \%$ & $0.00 \%$ & $92.80 \%$ \\
$18 S$ & BioClim & 184 & $7.30 \%$ & $0.00 \%$ & $92.70 \%$ \\
$18 S$ & Soil Properties & 180 & $15.30 \%$ & $0.00 \%$ & $84.70 \%$ \\
$18 S$ & Vegetation & 169 & $18.50 \%$ & $0.00 \%$ & $81.50 \%$ \\
CO1 & Location & 184 & $0.11 \%$ & $0.22 \%$ & $99.60 \%$ \\
CO1 & Habitat & 156 & $1.86 \%$ & $0.00 \%$ & $98.10 \%$ \\
CO1 & Topography & 184 & $3.30 \%$ & $0.46 \%$ & $96.20 \%$ \\
CO1 & BioClim & 184 & $12.00 \%$ & $0.00 \%$ & $88.00 \%$ \\
CO1 & Vegetation & 169 & $18.20 \%$ & $0.31 \%$ & $81.10 \%$ \\
CO1 & Soil Properties & 180 & $18.60 \%$ & $0.00 \%$ & $81.30 \%$ \\
FITS & Topography & 184 & $0.69 \%$ & $0.55 \%$ & $98.70 \%$ \\
FITS & Location & 184 & $0.93 \%$ & $0.38 \%$ & $98.20 \%$ \\
FITS & Habitat & 156 & $2.24 \%$ & $0.37 \%$ & $97.10 \%$ \\
FITS & BioClim & 184 & $18.50 \%$ & $0.00 \%$ & $80.40 \%$ \\
FITS & Soil Properties & 180 & $22.40 \%$ & $0.00 \%$ & $77.50 \%$ \\
FITS & Vegetation & 169 & $32.40 \%$ & $1.05 \%$ & $66.40 \%$ \\
PITS & Location & 184 & $0.03 \%$ & $0.00 \%$ & $100.00 \%$ \\
PITS & BioClim & 184 & $1.30 \%$ & $0.00 \%$ & $98.70 \%$ \\
PITS & Habitat & 156 & $2.16 \%$ & $0.00 \%$ & $97.80 \%$ \\
PITS & Topography & 184 & $2.98 \%$ & $0.03 \%$ & $96.90 \%$ \\
PITS & Soil Properties & 180 & $4.23 \%$ & $0.00 \%$ & $95.70 \%$ \\
PITS & Vegetation & 169 & $9.00 \%$ & $0.00 \%$ & $91.00 \%$ \\
\hline & & & & &
\end{tabular}




\section{$1160 \quad$ Figure legends}

\section{Figure 1}

1162 Figure 1 Map of $\mathbf{2 7 8}$ sites included in this study and illustration of taxonomic entries

1163 recovered with five metabarcodes. (A) Study area (gray shade) is defined within the State of

1164 California, United States. Sample sites are colored by three transect designations: coast (red),

1165 forest (blue) and shrub (green). Size of the points corresponds to the number of samples taken in

1166 the same area. Shape of the points (circle or triangle) represents areas within and outside of the

1167 University of California's Natural Reserve System (UCNRS, yellow shade, area size not to scale

1168 for visibility). Circles are within UCNRS, triangles are not. (B) Read abundance is grouped by

1169 the phylum they belong to after taxonomy assignment and decontamination for five

1170 metabarcodes targeted Bacteria and Archaea (16S), Eukaryota (18S), Metazoa (CO1), Fungi

1171 (FITS) and Viridiplantae (PITS). Only the most abundant 10 phyla are plotted for each

1172 metabarcode. All other phyla are summarized in the "Others" category. (C) Heatmap shows each

1173 metabarcode's taxonomic specificity. The results from each metabarcode (16S, 18S, CO1, FITS,

1174 PITS) are represented from inner to outer rings. Lighter color in one cell represents more

1175 taxonomic entries were recovered by that metabarcode for that phylum, gray color represents no

1176 entries. Phyla are indicated on the periphery. Background color of each pie wedge denotes the

1177 superkingdom (Red: Archaea, Blue: Eukaryota, Green: Bacteria, No background: Unknown) to

1178 which the phyla belonged at the time of taxonomy assignment (taxonomy file downloaded from

1179 NCBI at January 19, 2018). For eukaryotic phyla, Fungi (solid), Metazoa (dashed) and

1180 Viridiplantae (dotted) kingdoms are marked by different line types in an orange outline. 


\section{Figure 2}

1182 Figure 2 Boxplots of Shannon Index alpha diversity binned by different categories for the

1183 fungal ITS1 'FITS' metabarcode. The Shannon diversity Index for rarified FITS metabarcode

1184 is grouped by (A) location, (B) transect designation, (C) major habitat designation and (D)

1185 substrate type designation. Horizontal notch stands for the medians. Lower and upper box ranges

1186 (hinge) represent the 25 th and 75 th percentiles. The whiskers extend to data points no more than

$11871.5 *$ IQR (inter-quartile range) from the hinges. All data points are plotted additionally as circles

1188 using geom_jitter. P-values from Kruskal-Wallis tests on mean alpha diversity metrics across

1189 categories are denoted at the bottom of each panel. Post-hoc pairwise Dunn Test significance is

1190 denoted as $* \mathrm{P}<0.05, * * \mathrm{P}<0.01, * * * \mathrm{P}<0.001$. Bonferroni Adjusted $\mathrm{P}$ values are in Table S7.

$1191 \quad$ Figure 3

1192 Figure 3 Beta diversity plots based on Jaccard dissimilarity. The first two principal

1193 coordinates are plotted with percentage of variance explained included in axis label. We show

1194 selected Principal Coordinate Analysis (PCoA) plots from (A) $16 S$ and $18 S$ for major habitat. For

1195 comparison, (B) shows a partial constrained analysis of proximities (CAP) of community

1196 composition by major habitat while excluding the location effect. The first two axes are plotted

1197 for each metabarcode. Each point stands for a sampling site. The " $\mathrm{X}$ " marks the position of each

1198 group centroid. Major habitats still substantially overlap in (B) compared to (A) for $16 S$. Tree-

1199 dominated and herbaceous plant-dominated habitats show little overlap compared to (A) for $18 S$.

1200 (C) Re-analysis of (A) but with coastal sites removed shows the aquatic and herbaceous-

1201 dominated space is greatly reduced. (D) Selected PCoA plots grouped by transect designations,

1202 showing samples belonging to coastal sites account for the greater amount of variation in PCoA

1203 Axis. 1. (E) Selected PCoA plots grouped by national land cover classification, showing 
1204 developed space frequently overlap with other natural areas. Examples are for CO1 and PITS. (F)

1205 Selected PCoA plots for FITS and PITS showing fungal beta diversity in sand is low compared to

1206 other substrates and compared to beta diversity for non-fungal taxa.

1207 Figure 4

1208 Figure 4 PCoA based on Jaccard dissimilarity with samples grouped by minor habitat and

1209 plotted within four major habitat categories. Results of PERMANOVA and beta dispersion

1210 show significant results $(\mathrm{P}<0.001$, number of permutations $=2999$; Table $\mathrm{S} 10.5-6)$ in minor

1211 habitat classification. Some minor habitat groups separate while others overlap, and patterns of

1212 compositional similarity (overlap) are different for different metabarcodes: (A) 16S, (B) $18 S$, (C)

1213 CO1, (D) FITS, (E) PITS.

1214 Figure 5

1215 Figure 5 Gradient forest result for filtered CALeDNA dataset. (A) Ranked overall

1216 importance for 33 environmental predictors. (B) Ranked goodness-of-fit (1 - Relative Error rates)

1217 for the top 30 families (response variables). (C) and (D) show the community turnover along the

1218 three most important environmental gradients: elevation, sand percentage and photosynthetic

1219 activity proxy (NDVI32). (C) The gray histogram shows binned split importance at each gradient.

1220 Kernel density of splits (black lines), of observed predictor values (red lines) and of splits

1221 standardized by observation density (blue lines) are overlaid. The horizontal dashed line

1222 indicates where the ratio is 1 . Each curve integrates to the importance of the predictor. (D) The

1223 line shows cumulative importance distributions of splits improvement scaled by $\mathrm{R}^{2}$ weighted

1224 importance and standardized by density of observations, averaged over all families. 


\section{Figure 6}

1226 Figure 6 Map of transformed environmental variables following gradient forest predictions of

1227 biodiversity turnover from eDNA results (A) compared with uninformed, standardized

1228 environmental variables (B) and current major ecoregion maps (C-E) in California. The map

1229 shows the first three principal dimensions of biologically predicted (A) or uninformed (B)

1230 community compositions with an RGB color palette with $100 \mathrm{~m}$ resolution. The biplot of the first

1231 two PCs of the transformed environment space with (inset A) or without (inset B) biological

1232 information provides a color key for the compositional variation $(\mathrm{n}=50 \mathrm{k})$. Similar colors

1233 approximate similar community in the transformed environmental space. The gray crosses

1234 denote the input eDNA sites $(n=272)$. Vectors denote the direction and magnitude of eight most

1235 important environmental correlates. (C-E) Selected major ecoregions maps are provided for

1236 comparisons with the gradient forest map (A). (C) EPA Level II Ecoregions of North America

1237 (U.S. Environmental Protection Agency 2010). (D) EPA Level III Ecoregions of California (U.S.

1238 Environmental Protection Agency 2012). (E) USDA Ecoregion Sections in California (USDA

1239 Forest Service 2007).

$1240 \quad$ Figure 7

1241 Figure 7 eDNA-based ecological co-occurrence network and relationship with gradient

1242 forest model goodness-of-fit $\mathbf{R}^{\mathbf{2}}$. (A) 369 families (as nodes) are included in the network and

1243290 of those have at least 1 edge connecting them to another node. Colors are scaled by degrees

1244 for nodes and betweenness centrality for edges. Lower values have warmer colors. Node labels

1245 are families but are not intended to be legible in print. (B) OLS linear regression and quantile-

1246 quantile plot showing the interaction between network sum of degrees and frequency of taxa in

1247 sample sites with the dependent variable of gradient forest Family goodness-of-fit $\mathrm{R}^{2}$. There 
1248 were 304 families included as joint observations in gradient forest and network results. The

1249 adjusted $\mathrm{R}^{2}=0.22$, network sum estimate $=0.01(\mathrm{t}$-value $=5.44 ; \mathrm{p}=0.00)$, frequency in sites

1250 estimate $=0.00(\mathrm{t}$-value $=0.18 ; \mathrm{p}=0.86)$, and interaction between network sum and frequency in

1251 sites $=0.00(\mathrm{t}$-value $-2.38 ; \mathrm{p}=0.02)$. (C) Phylogenetic tree made with the Open Tree of Life

1252 targeting input families as tips. Heatmap labels correspond to the range of gradient forest $\mathrm{R}^{2}$

$1253(0.078-0.913)$ from yellow to dark green, and to the range of network degrees (0-48) from yellow

1254 to purple. Families too rare to be included in the network analysis (in fewer than 28 sites) are not

1255 colored in heatmaps. Arrows indicate the following clades: brown $=$ fungi, mustard $=$

1256 Enterobacteriaceae, blue $=$ Flavobacteriia, green $=$ Streptophyta, red $=$ SAR supergroup. 
bioRxiv preprint doi: https://doi.org/10.1101/2020.06.19.160374; this version posted June 20, 2020. The copyright holder for this preprint (which was not certified by peer review) is the author/funder. All rights reserved. No reuse allowed without permission.

\section{$1258 \quad$ Figures}

\section{Figure 1}
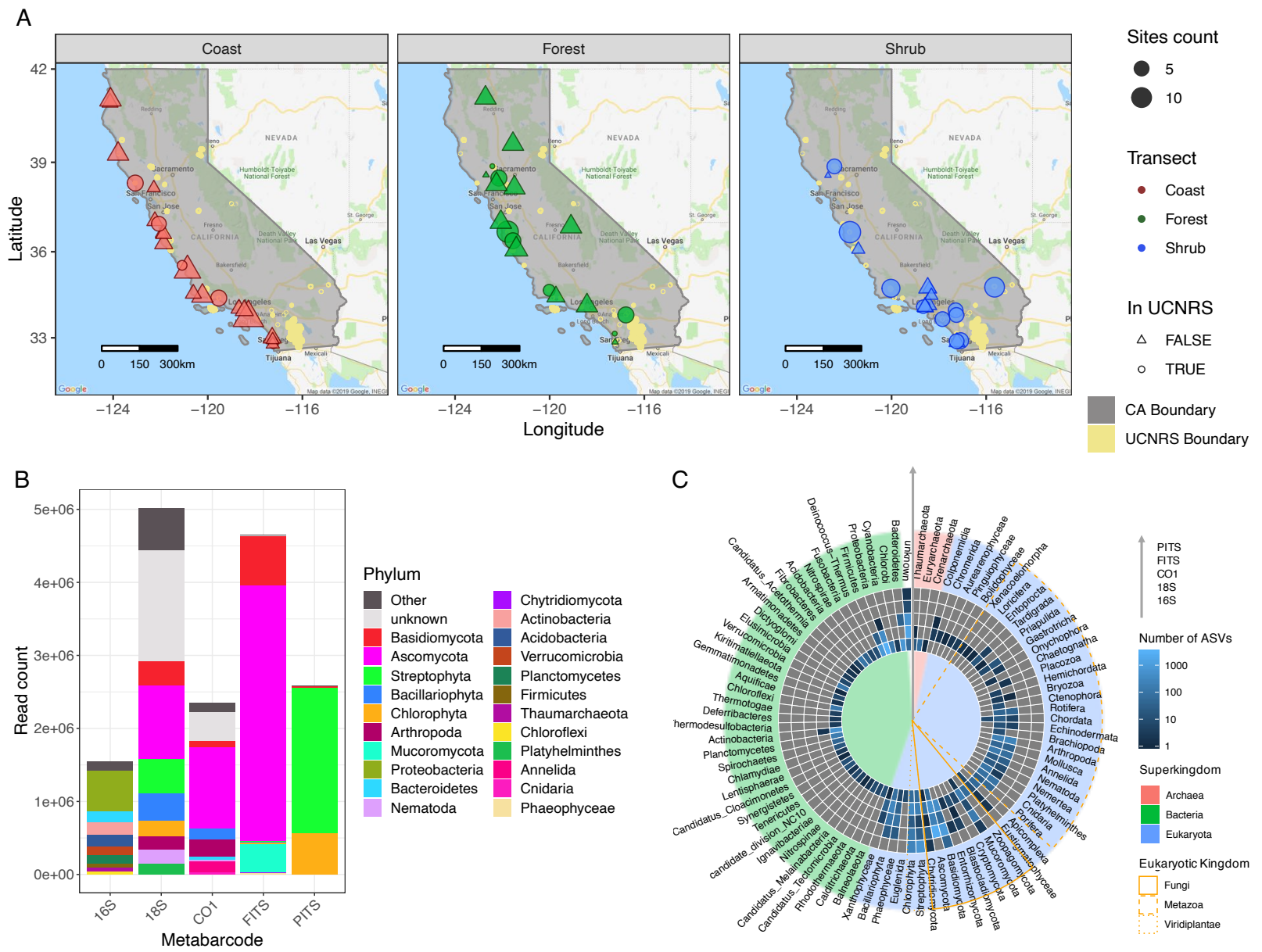
bioRxiv preprint doi: https://doi.org/10.1101/2020.06.19.160374; this version posted June 20, 2020. The copyright holder for this preprint (which was not certified by peer review) is the author/funder. All rights reserved. No reuse allowed without permission.

\section{Figure 2}
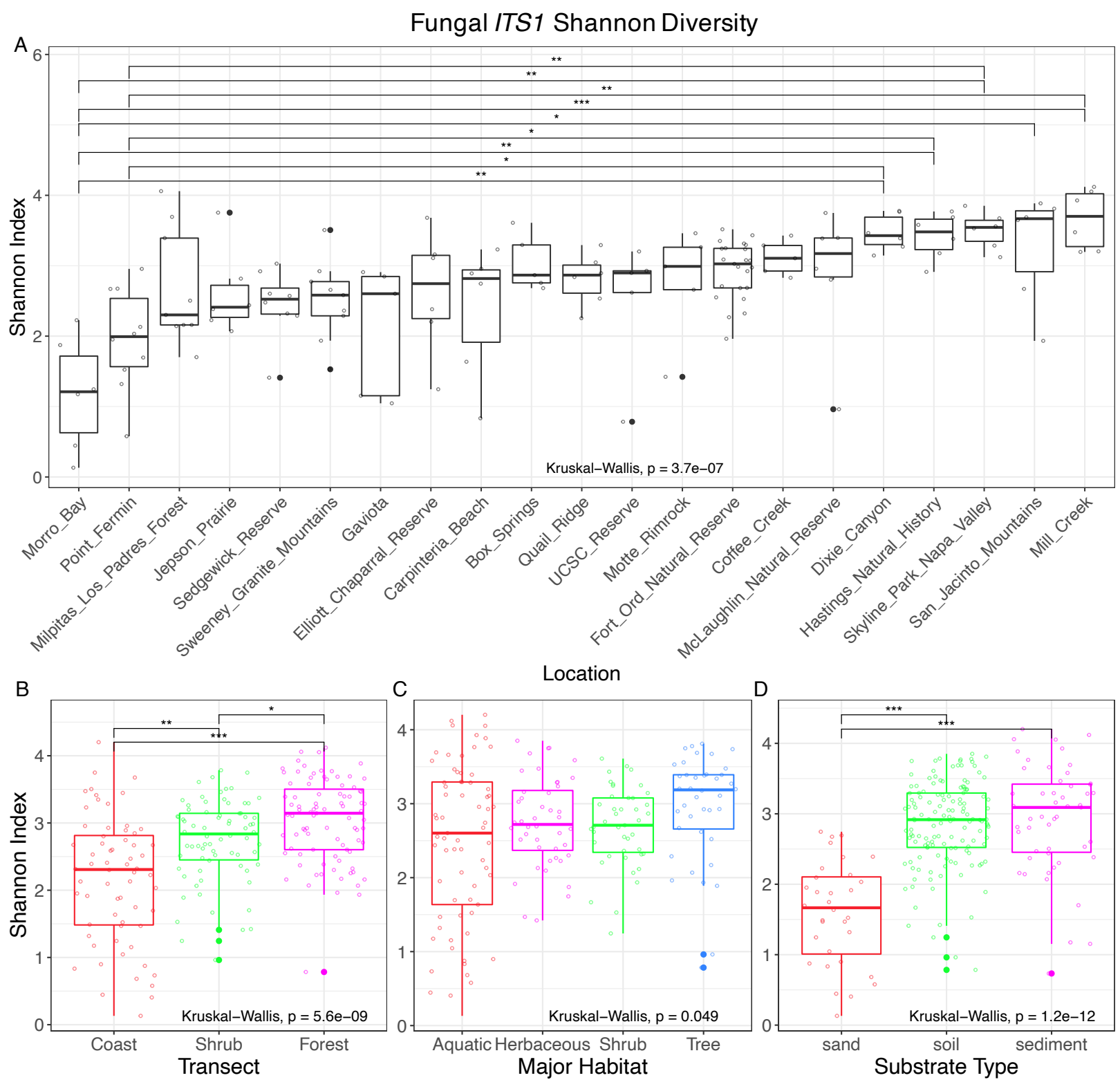
bioRxiv preprint doi: https://doi.org/10.1101/2020.06.19.160374; this version posted June 20, 2020. The copyright holder for this preprint (which was not certified by peer review) is the author/funder. All rights reserved. No reuse allowed without permission.

\section{$1265 \quad$ Figure 3}
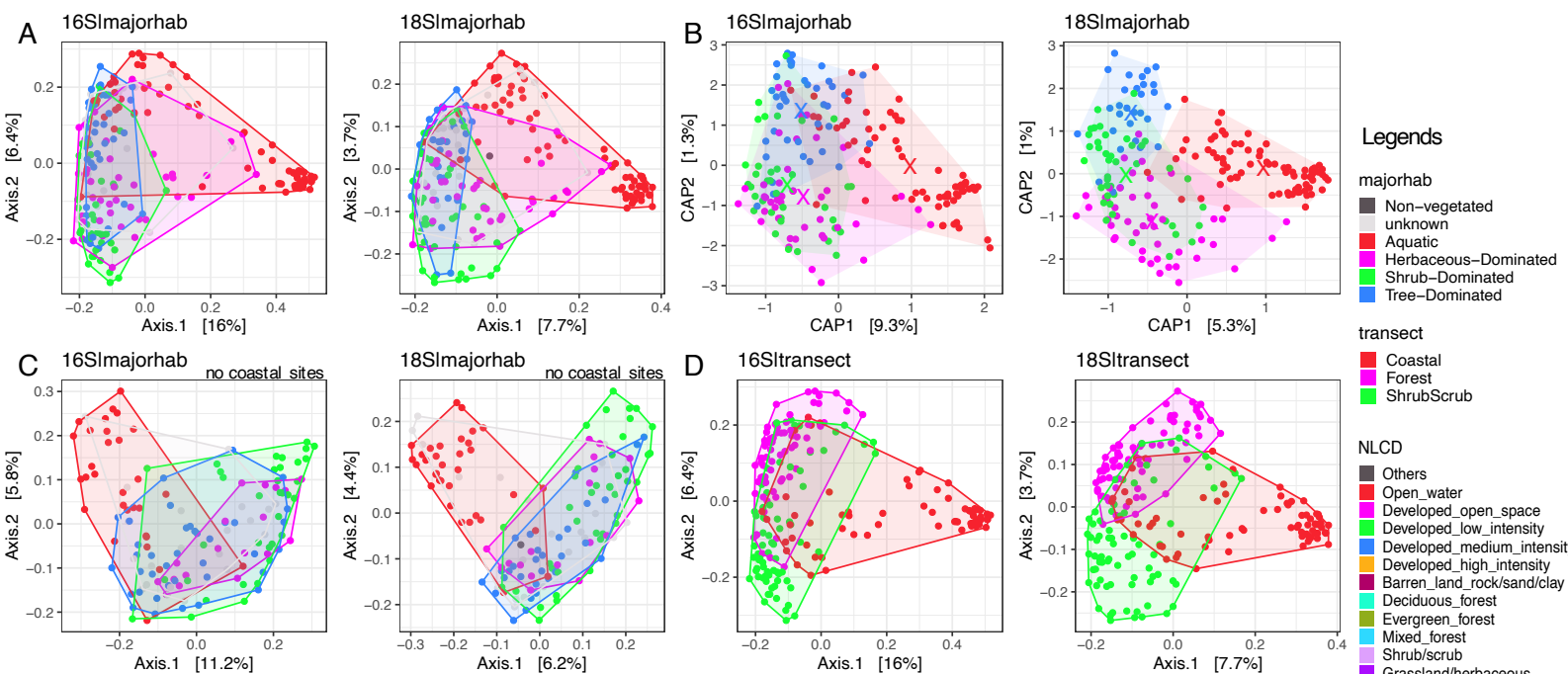

Coastal

ShrubScrub

E CO1INLCD
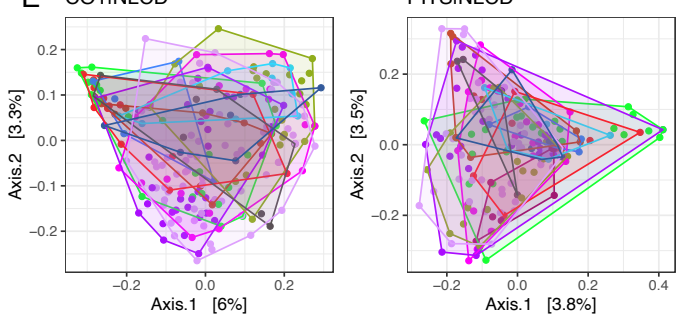

F FITSISOS

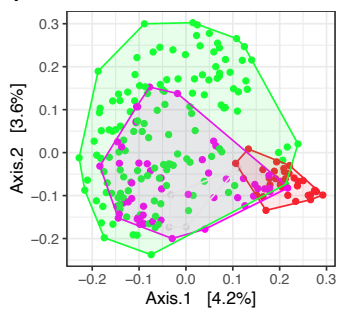

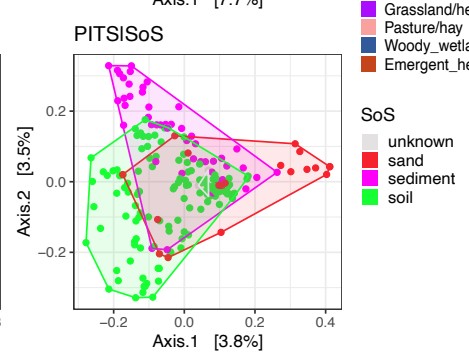


bioRxiv preprint doi: https://doi.org/10.1101/2020.06.19.160374; this version posted June 20, 2020. The copyright holder for this preprint (which was not certified by peer review) is the author/funder. All rights reserved. No reuse allowed without permission.

\section{Figure 4}
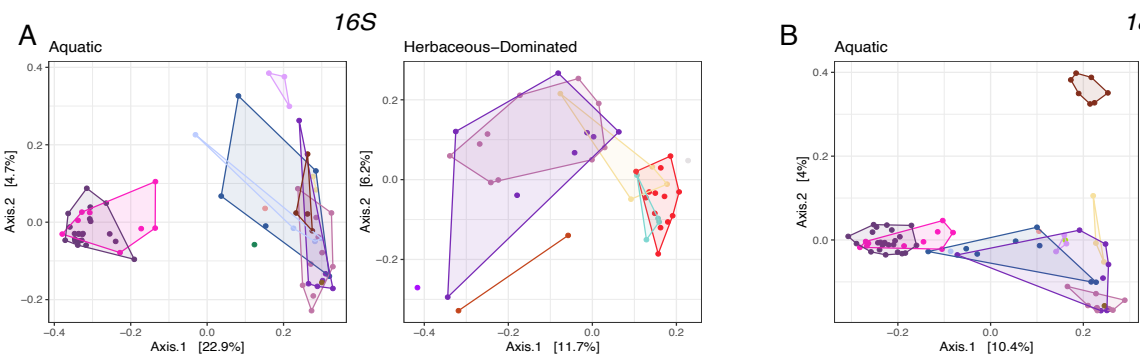

$18 S$
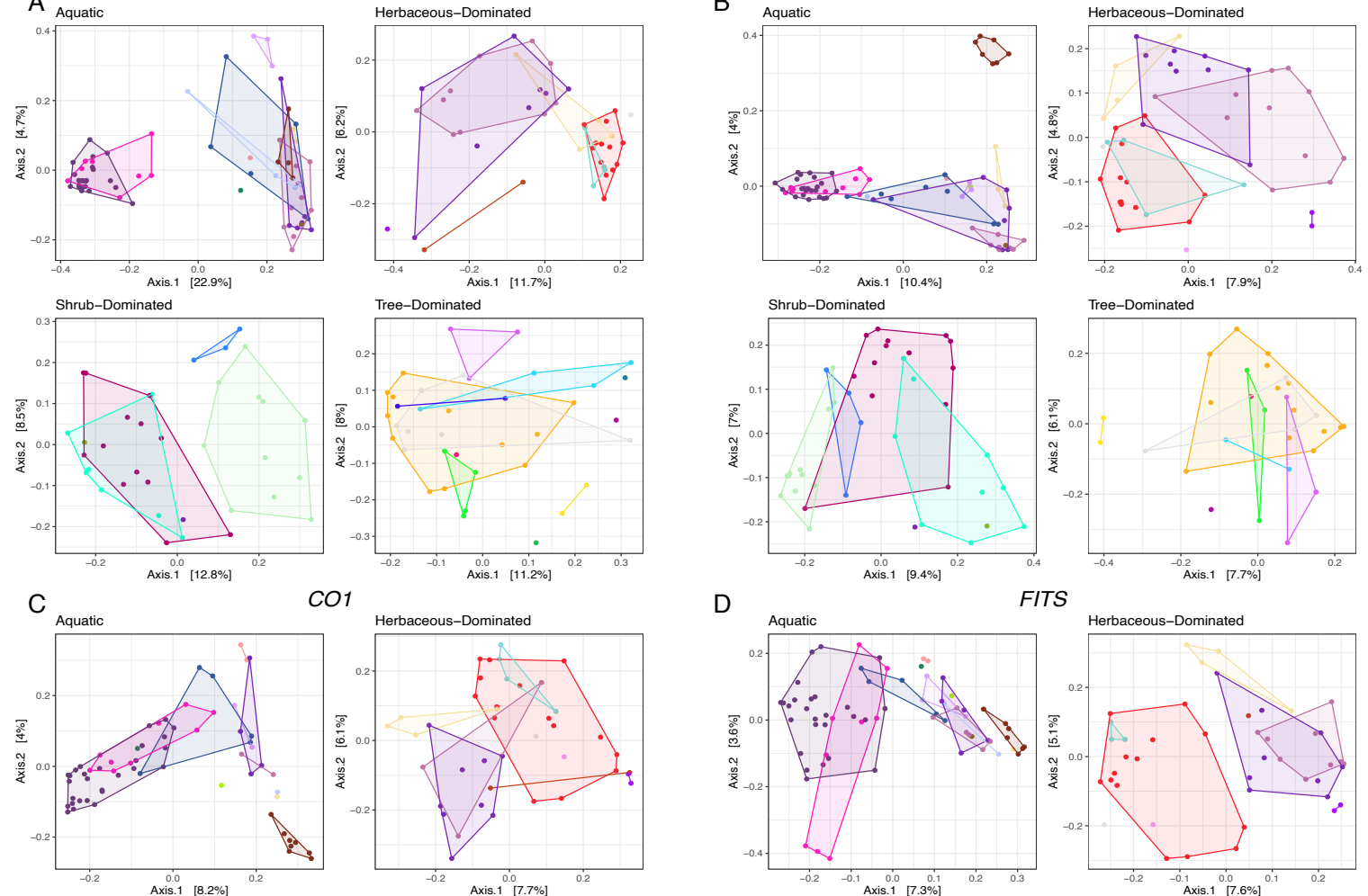

CO1 Herbaceous-Dominated
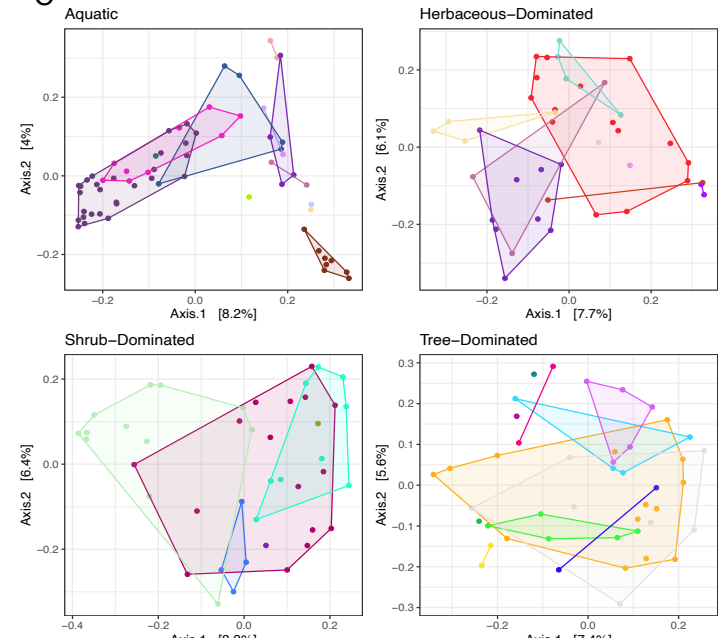

D
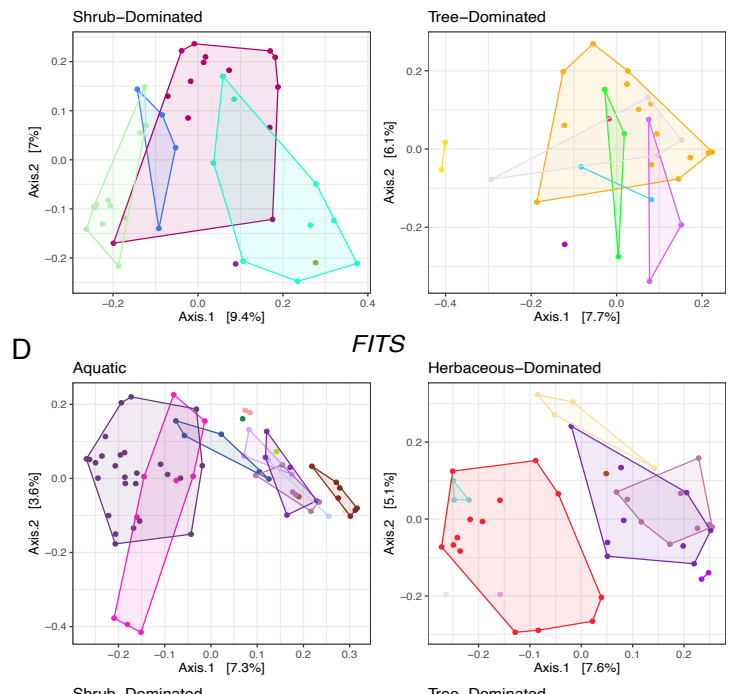

Herbaceous-Dominated
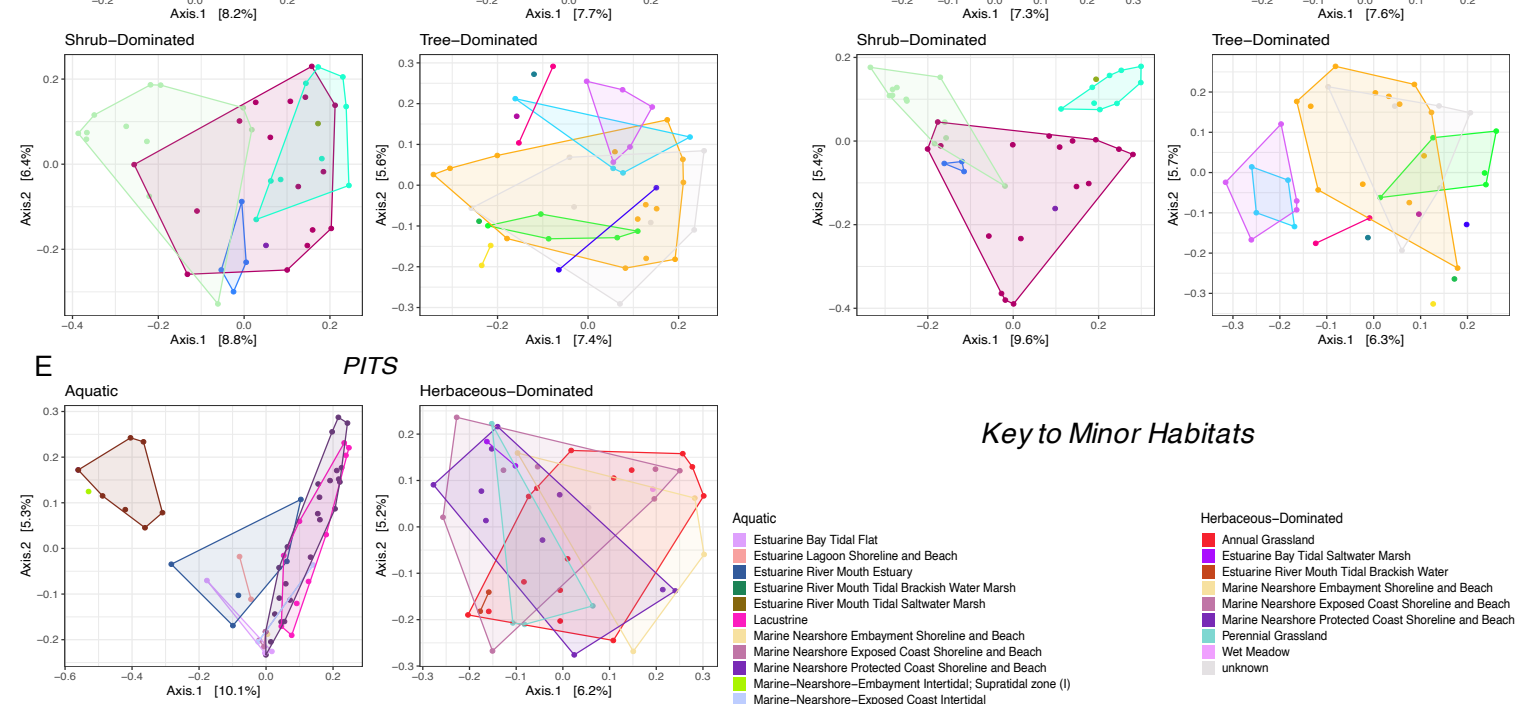

Herbaceous-Dominated
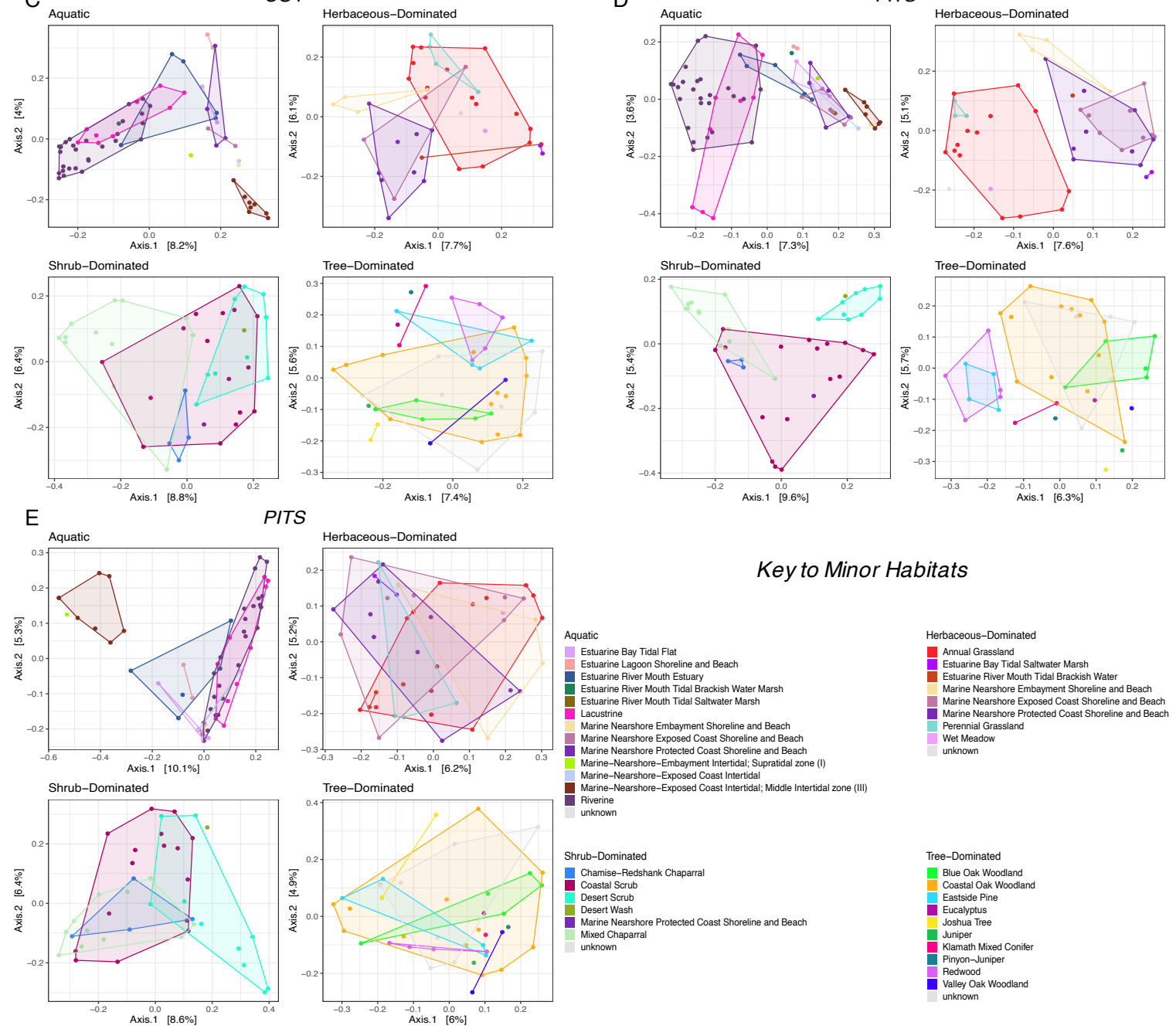

1269 
bioRxiv preprint doi: https://doi.org/10.1101/2020.06.19.160374; this version posted June 20, 2020. The copyright holder for this preprint (which was not certified by peer review) is the author/funder. All rights reserved. No reuse allowed without permission.

Figure 5
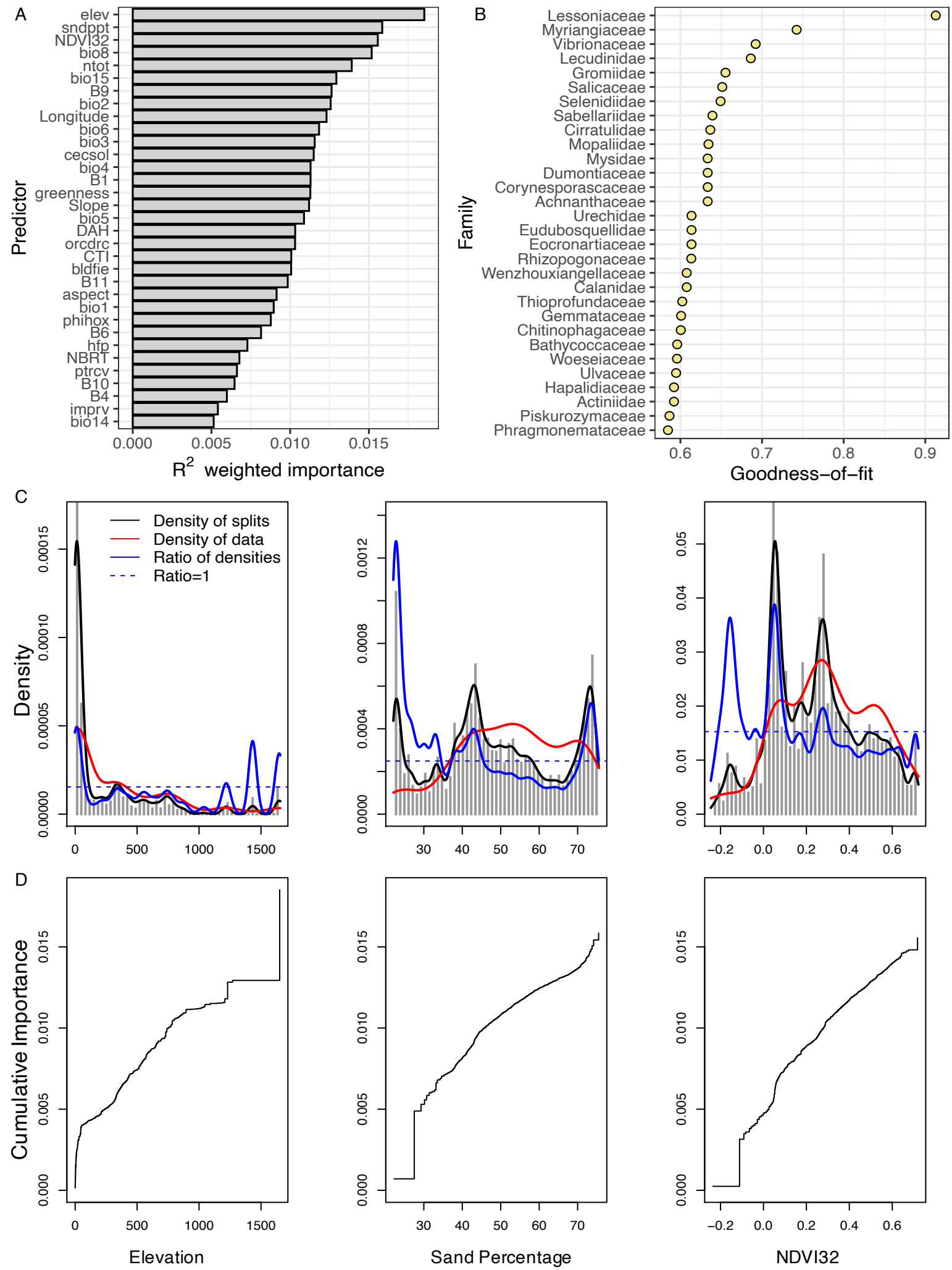

1271 
bioRxiv preprint doi: https://doi.org/10.1101/2020.06.19.160374; this version posted June 20, 2020. The copyright holder for this preprint (which was not certified by peer review) is the author/funder. All rights reserved. No reuse allowed without permission.

\section{$1272 \quad$ Figure 6}
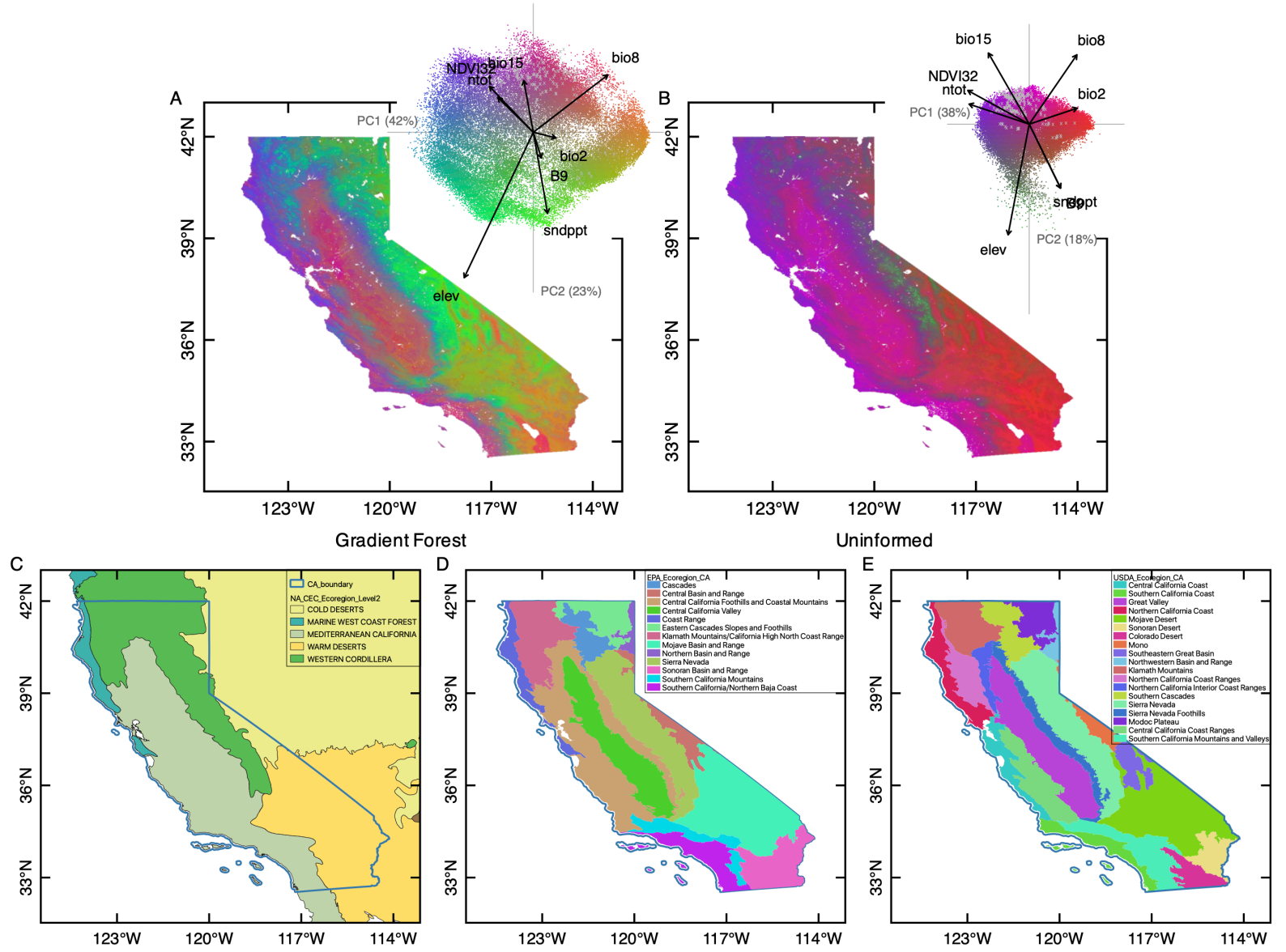
bioRxiv preprint doi: https://doi.org/10.1101/2020.06.19.160374; this version posted June 20, 2020. The copyright holder for this preprint (which was not certified by peer review) is the author/funder. All rights reserved. No reuse allowed without permission.

\section{Figure 7}
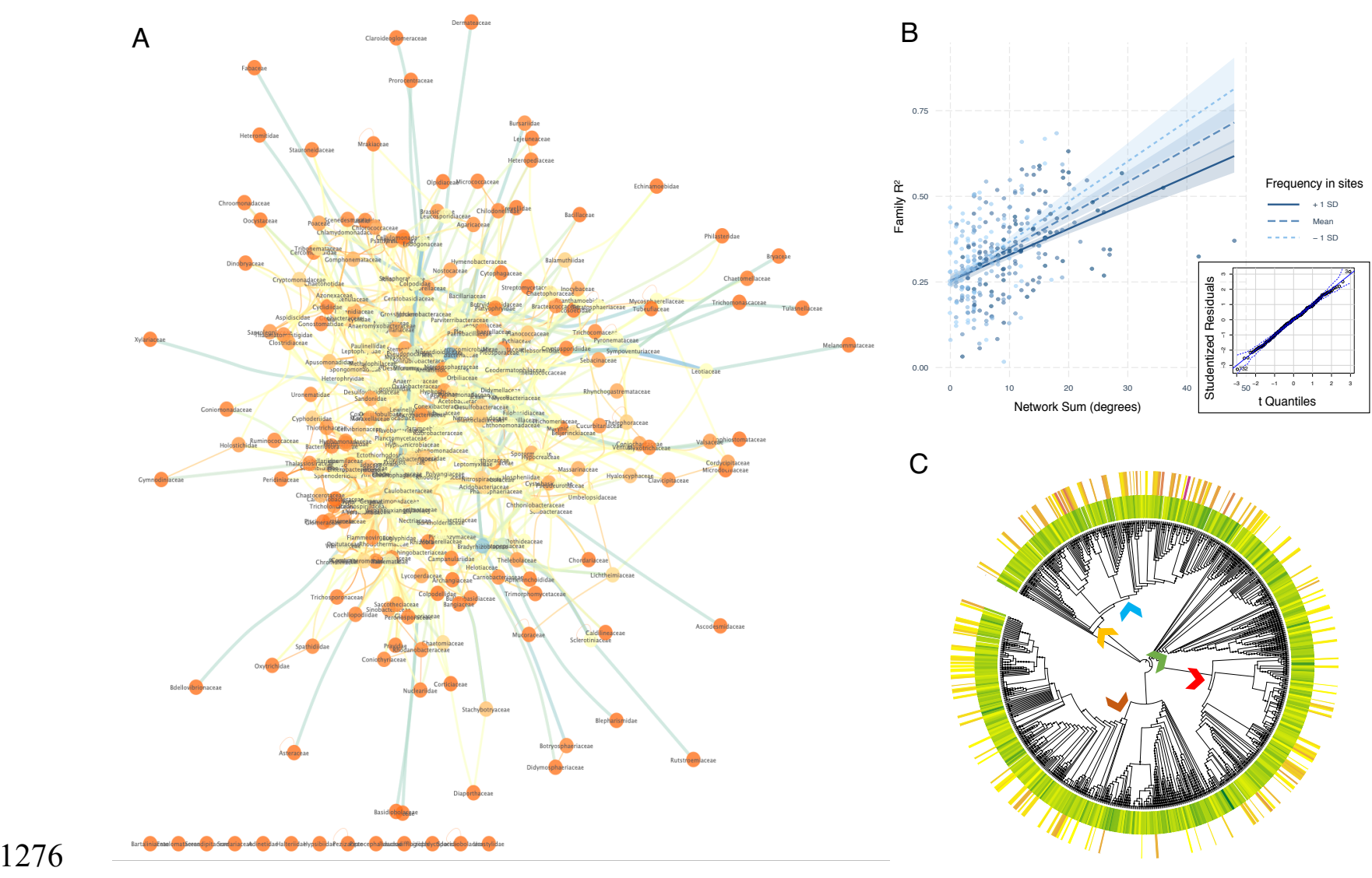

C

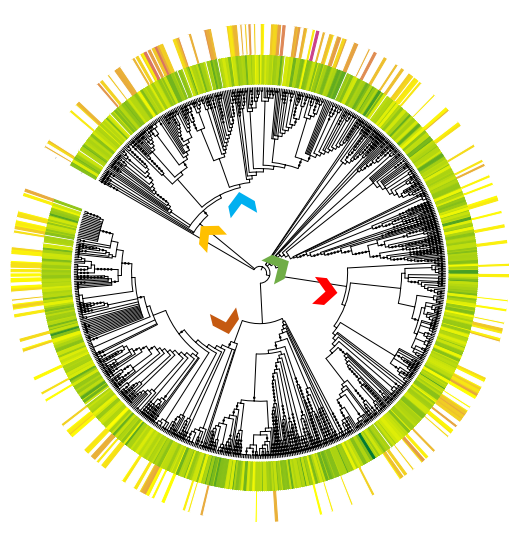

